\title{
GPU Accelerated Path Tracing of Massive Scenes
}

\author{
MILAN JAROŠ, LUBOMÍR ŘÍHA, PETR STRAKOŠ, and MATĚJ ŠPEŤKO,
}

IT4Innovations, VSB-Technical University of Ostrava, Czech Republic
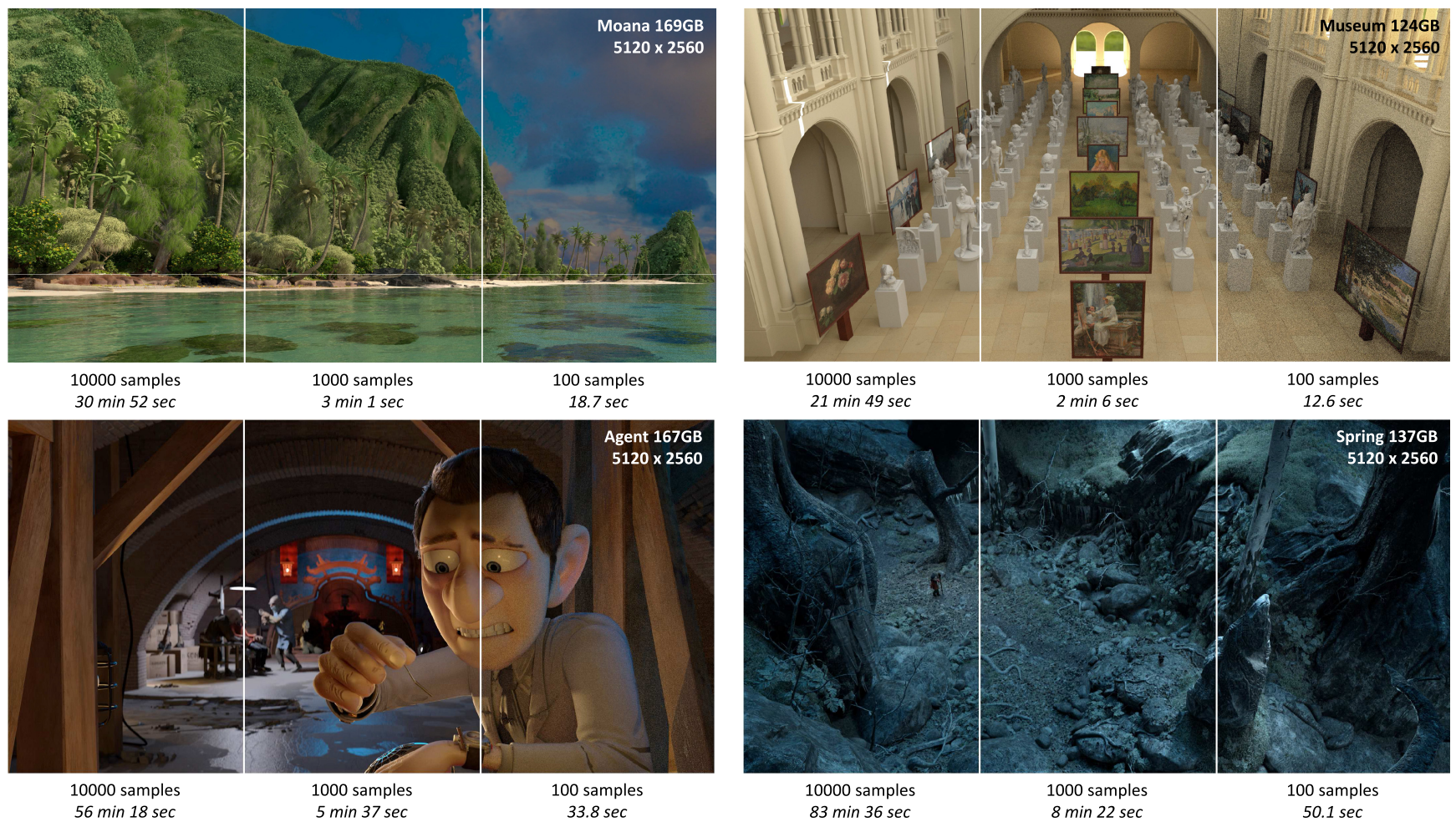

Fig. 1. Examples of rendering of massive scenes using our modified version of Blender Cycles path tracer. The rendering time is for the different number of samples per pixel measured on an NVIDIA DGX-2 machine with 16 GPUs. All the scene data, including geometry, were partially distributed among the memories of all GPUs. No noise reduction filter was applied.

This article presents a solution to path tracing of massive scenes on multiple GPUs. Our approach analyzes the memory access pattern of a path tracer and defines how the scene data should be distributed across up to 16 GPUs with minimal effect on performance. The key concept is that the parts of the scene that have the highest amount of memory accesses are replicated on all GPUs.

This work was supported by The Ministry of Education, Youth, and Sports from the Large Infrastructures for Research, Experimental Development, and Innovations project "e-Infrastructure CZ-LM2018140."

Authors' address: M. Jaroš, L. Ř́ha, P. Strakoš, and M. Špetko, IT4Innovations, VSB Technical University of Ostrava, 17. listopadu 2172/15, 70800 Ostrava-Poruba, Czech Republic; emails: \{milan.jaros, lubomir.riha, petr.strakos, matej.spetko\}@vsb.cz.

Permission to make digital or hard copies of all or part of this work for personal or classroom use is granted without fee provided that copies are not made or distributed for profit or commercial advantage and that copies bear this notice and the full citation on the first page. Copyrights for components of this work owned by others than ACM must be honored. Abstracting with credit is permitted. To copy otherwise, or republish, to post on servers or to redistribute to lists, requires prior specific permission and/or a fee. Request permissions from permissions@acm.org.

(C) 2021 Association for Computing Machinery.

0730-0301/2021/04-ART16 \$15.00

https://doi.org/10.1145/3447807
We propose two methods for maximizing the performance of path tracing when working with partially distributed scene data. Both methods work on the memory management level and therefore path tracer data structures do not have to be redesigned, making our approach applicable to other path tracers with only minor changes in their code. As a proof of concept, we have enhanced the open-source Blender Cycles path tracer.

The approach was validated on scenes of sizes up to $169 \mathrm{~GB}$. We show that only $1-5 \%$ of the scene data needs to be replicated to all machines for such large scenes. On smaller scenes we have verified that the performance is very close to rendering a fully replicated scene. In terms of scalability we have achieved a parallel efficiency of over $94 \%$ using up to 16 GPUs.

CCS Concepts: • Computing methodologies $\rightarrow$ Computer graphics; Ray tracing; Distributed algorithms;

Additional Key Words and Phrases: Multi GPU path tracing, NVLink, CUDA unified memory, data distributed path tracing, distributed shared memory path tracing 


\section{ACM Reference format:}

Milan Jaroš, Lubomír Říha, Petr Strakoš, and Matěj Špet'ko. 2021. GPU Accelerated Path Tracing of Massive Scenes. ACM Trans. Graph. 40, 2, Article 16 (April 2021), 17 pages.

https://doi.org/10.1145/3447807

\section{INTRODUCTION}

In this article, we present a solution for path tracing of massive scenes by combining the performance and, most importantly, memory of multiple GPUs. The proposed approach is applicable to production rendering. In recent years, the advances in GPU technology made GPU rendering popular for its superior speed over CPU rendering [Maxon 2019]. However, the significant drawback of GPUs when compared to CPUs is the limited memory size. This is one of the key reasons why CPUs still play a dominant role in production rendering as described by authors of the major rendering systems used in film production [Burley et al. 2018; Christensen et al. 2018; Fascione et al. 2018; Georgiev et al. 2018; Kulla et al. 2018]. For instance, Christensen et al. [2018] show that scenes from Pixar's Coco movie were using up to 120 GB. Scenes of such sizes do not fit into the memory of a single GPU.

Our main technical contribution is a solution to this problem that is based on replication of a small amount of scene data, between $1 \%$ and $5 \%$, and well-chosen distribution of the rest of the data into the memory of several GPUs. Both replication and distribution of data is based on a memory access pattern analysis of a path tracer during a 1spp prepass. The data with the highest number of memory accesses are replicated and the rest is stored only in the memory of the GPU that had the highest number of accesses to it. This minimizes the penalty associated with reading data from the remote memory and is effective at providing near-linear scalability. To the best of our knowledge, this article presents the first work describing multi-GPU-based path tracing of massive scenes where a major part, up to $99 \%$, of scene data is split up among the memories of up to 16 GPUs.

Our second main contribution is the demonstration that our proposed approach works on a memory management level. This means that any path tracing code that supports GPU acceleration using CUDA can adopt our approach without redesigning its internal data structures. As a proof of concept, the proposed mechanism was implemented in the complex open-source Blender Cycles path tracing engine [Blender Foundation 2018].

Our approach relies on two key technologies: (i) NVLink GPU interconnect, which enables multiple GPUs to efficiently share the content of their memories due to its high bandwidth and low latency, [Li et al. 2020] and (ii) CUDA Unified Memory (UM), which provides programmers with control over data placement across the memories of interconnected GPUs [Harris 2017].

Today, in the professional computer graphics sector, only two GPUs can be connected using NVLink. However, in the world of High-performance Computing (HPC) NVLink is already used to interconnect up to 16 GPUs [NVIDIA 2018c] as in the case of NVIDIA DGX-2, which has 512 GB of shared GPU memory [NVIDIA 2019]. We expect that it is merely a matter of a few years before servers like this will be equipped with GPUs with hardware acceleration for path tracing such as Ray Tracing (RT) cores [NVIDIA 2018d].
The article is organized as follows. Section 2 describes the state of the art in the field. Section 3 gives basic information about Blender Cycles and our modifications in the path tracer to support multi-GPU rendering. It also describes hardware platforms and scenes used for benchmarking. Section 4 describes the proposed methods together with analysis of the memory access patterns during the path tracing. This section also shows how different aspects of data management in memory affects the performance. Section 5 provides a performance and scalability evaluation on massive scenes. Finally, Section 6 provides a summary of the article and our future plans in this area.

\section{RELATED WORK}

The majority of film production rendering systems rely on path tracing. These systems almost exclusively use CPUs to deliver the final image [Burley et al. 2018; Christensen et al. 2018; Fascione et al. 2018; Georgiev et al. 2018; Kulla et al. 2018] as their scenes are too large to fit in the limited memory of a GPU. There are two possible solutions for the limited local memory: (i) out-of-core rendering and (ii) distributed or parallel rendering. We discuss both approaches, but more attention is given to the distributed rendering, since our proposed solution belongs to this area.

\subsection{Out-of-core Rendering}

The GPU accelerated rendering of large scenes is mostly handled using out-of-core techniques. Budge et al. [2009] presented an approach that dealt with out-of-core data management for heterogeneous architectures consisting of multiple CPU/GPU nodes. Son et al. [2017] addressed the problem of path tracing on various sets of device configurations. Their approach supported out-of-core rendering for heterogeneous clusters with CPU/GPU nodes and network interconnection.

In terms of multi-GPU rendering, Zhou et al. [2009] presented a photorealistic rendering system that used an out-of-core approach for textures together with dynamic scheduling for efficient parallelization. As it implemented REYES rendering on GPUs [Cook et al. 1987] it mainly contributed to rasterization rather than path tracing. Pantaleoni et al. [2010] proposed a rendering system capable of fast lighting iterations over large scenes. It used an out-ofcore GPU ray tracing algorithm for the computation of directional occlusion and spherical integrals. Wang et al. [2013] suggested a GPU rendering framework that can handle massive scenes with out-of-core geometry and complex lighting. The solution was designed to use only one GPU and applied a many-lights method instead of path tracing.

\subsection{Distributed Rendering}

There is a large body of work on distributed rendering techniques for massive scenes on distributed memory systems. In the standard classification proposed by Molnar et al. [1994] these works fall into one of these categories: sort-first, which results in imagebased partitioning; sort middle, related to rasterization only; and sort-last, which uses scene data distribution.

2.2.1 Image-Parallel Rendering. Sort-first methods are based on screen-partitioning, and the workload is distributed among processors or machines per blocks of pixels of a rendered image. In the 
most common (and also the most efficient) way the scene data is fully replicated in all local memories, and ray tracing is embarrassingly parallel. In the case of ray tracing complex scenes that do not fit into local memory, this approach results in on-demand scene data movement while rays remains fixed [Demarle et al. 2005; Keller et al. 2017; Parker et al. 1998; Wald et al. 2003]. Our proposed solution is based on scene data communication while rays never leave the GPU they are created on.

2.2.2 Data-Parallel Rendering. Sort-last methods, also called data-parallel, distribute the workload by subdividing the scene data. In the case of distributed ray tracing, these approaches transfer ray data among processors or machines, while scene data do not move after the initial distribution. Kato et al. [2001] used this approach for production rendering on a cluster of workstations. More recently this approach was used in the field of scientific visualizations of massive data sets from scientific simulations on supercomputers [Usher et al. 2019]. Navratil et al. (2014, 2012) proposed a hybrid (combination of image-parallel and data-parallel) approach for distributed memory supercomputers that used dynamic ray scheduling. The proposed dynamic solutions worked well, except in situations where ray communication costs exceeded data load costs such as in high-quality rendering at high resolution.

\subsection{Distributed Shared Memory Systems}

During the late 1990s Shared Memory Processors (SMP) with large amounts of Distributed Shared Memory (DSM) became commercially available [Protic et al. 1995]. In such machines, each processor has a local memory with caches, and a hardware or software layer transparently creates an illusion of global shared memory for applications [Protic et al. 1996]. As multiple processors read and write from/to one shared memory, the key problem is preserving a coherent view of shared data, which in practice is solved by various cache-coherent mechanisms [Hennessy et al. 1999].

DSM systems exhibit Non-Uniform Memory Access (NUMA), as the latency to access remote data is considerably larger than the latency to access local data. On such machines, good data locality is therefore critical for high performance. There were several methods that improved data locality over basic cache coherency using replication/migration techniques as summarised in Soundararajan et al. [1998]. This is also the key concept of our proposed approach.

In addition to data replication, the performance of DSM systems can also be improved by application-specific techniques to reduce memory bandwidth requirements. For ray tracing of incoherent rays, such a technique was proposed by Aila and Karras [2010].

In the past large DSM systems such as SGI Origin 2000, KSR1, and Stanford DASH were used for ray tracing of large scenes, such as in Parker et al. [1999], Keates and Hubbold [1994], and Singh et al. [1994], respectively. In these works, authors used screen partitioning, but proposed different solutions to work distribution, load balancing, and synchronization in the case of interactive rendering.

\subsection{CUDA Unified Memory for Multi-GPU Systems}

NVIDIA's Unified Memory (UM) [Harris 2017; Sakharnykh 2017b] manages communication between multiple GPUs and CPUs transparently by adopting DSM techniques. Alternative approaches have been also proposed [AlSaber and Kulkarni 2013; Gelado et al. 2010a; Jablin et al. 2012a]. UM simplifies both out-of-core processing between GPUs and CPUs as well as multi-GPU processing, ${ }^{1}$ and combinations of both. Previously, the applications focusing on large data processing on GPUs required algorithm-specific techniques for memory handling [Al-Saber and Kulkarni 2015; Gelado et al. 2010b; Huynh et al. 2012; Jablin et al. 2012b; Krizhevsky et al. 2012; Sabne et al. 2013; Seo et al. 2015; Shamoto et al. 2015].

In terms of HW technologies, NVlink interconnect is the key enabler of DSM multi-GPU systems. Li et al. [2020] provided thorough evaluation of several variants of NVLink interconnects against PCIe bus. Chien et al. [2020] evaluated the performance of advanced UM features such as prefetching and user controlled data placement [NVIDIA 2018a] on two different platforms, one with PCIe 3.0 interconnect between CPUs and GPUs and one with NVLink interconnect (based on Power9 CPU). A critical mechanism for UM is prefetching, page-eviction due to memory over subscription, and page migration between GPUs. The works of [Agarwal et al. 2015; Baruah et al. 2020; Ganguly et al. 2019, 2020; Young et al. 2018] proposed new algorithms to improve UM performance in the case of transparent memory management. In contrast, our approach controls the page placement and replication manually based on analysis of memory access patterns.

Several works from the CG area use UM on multi-GPU systems. Christensen et al. [2017] efficiently used NVLink for image composition after distributed rendering on up to 8 GPUs. Kim et al. [2017] utilized multiple GPUs for scalable split frame rendering (SFR), which assigns disjoint regions of a frame to different GPUs. Finally, Xie et al. [2019] used multi-GPU system for rendering for Virtual Reality systems. They exploited the data locality of scene objects to reduce inter-GPU memory traffic.

\section{BLENDER CYCLES PATH TRACER}

Blender has a production renderer called Cycles. It is an unbiased renderer based on unidirectional path tracing that supports CPU and GPU rendering. For ray tracing acceleration it uses a Bounding Volume Hierarchy (BVH). The BVH code is based on an implementation by NVIDIA [Aila and Laine 2009; Aila et al. 2012] with some additional code adaptation from Embree [Wald et al. 2014].

In terms of GPU rendering, Cycles supports CUDA, Optix, and OpenCL technology. CUDA and OpenCL implementations support the same features as a CPU one does, except for Open Shading Language (OSL) and Advanced Volume Light Sampling support. Optix support enables hardware acceleration of ray tracing on GPUs with RT cores, i.e., GPUs based on Turing architecture.

\subsection{Extensions for Multi-GPU Support}

In our previous work [Jaros et al. 2017], we extended the Cycles to support new parallel hardware architectures (e.g., Intel Xeon Phi co-processors and processors) and other HPC technologies (e.g., distributed rendering using MPI). We build on this extended Cycles version, although none of these extensions is needed for the work presented here. The original Cycles version can be used directly, since our approach uses only rendering routines for $\mathrm{x} 86 \mathrm{CPUs}$ and CUDA kernels for GPUs.

${ }^{1}$ All GPUs must be in a single server. 
To implement the proposed approach, only a few changes need to be made to the original GPU branch of Cycles. Most importantly, the core of the path tracing CUDA kernel used for the final rendering remains unchanged. The only modifications inside this kernel concern implementations of software counters that record memory access statistics. From a rendering point of view, the functionality of this kernel remains completely unchanged. Our modification splits each data structure used by the path tracer into chunks of a predefined size and counts the number of accesses per chunk. Since there is a performance overhead caused by this extra work it is recommended to have these modifications in a separate kernel.

The most significant changes are made to the CPU code to support the CUDA Unified Memory, which is used to both replicate or distribute selected chunks of scene data among multiple GPUs. This is done using cudaMallocManaged instead of cudaMalloc, together with cudaMemAdvise data placement hints introduced in CUDA 8.0. The CUDA UM mechanism is thoroughly described in [Chien et al. 2020; Ganguly et al. 2019; Gayatri et al. 2019]. For a shorter description, see Appendix C.

The overall workflow is as follows:

(1) distribute the data structures evenly among all GPUs,

(2) run the kernel with memory access counters and get the memory access statistics,

(3) redistribute the data structures among GPUs based on memory access statistics,

(4) run the original path-tracing kernel with redistributed data.

Our extended version of Cycles works as a distributed renderer that is able to render a single image using multiple servers with replicated scene data. It is in the form of a command line tool that uses Blender to preprocess a scene, generate a complete scene description (needed for path tracing), and save it into a file. This file is then passed to the Cycles as a command line parameter.

\subsection{Multi-GPU Benchmark Systems}

In this article, we use two multi-GPU platforms that can perform massive scene rendering. The first one is a BullSequana X410-E5 NVLink-V blade server [Atos 2017] with 4 Tesla V100 GPUs [NVIDIA 2017], each with 16 GB of memory and direct NVLink interconnect. The server is installed in the Barbora HPC cluster at IT4Innovations [2019] and therefore we will refer to it as Barbora.

The second, more advanced platform is NVidia DGX-2 [NVIDIA 2019], which is able to process massive scenes of sizes up to $512 \mathrm{~GB}$ in the shared memory of its 16 Tesla V100 GPUs, each with 32 GB of memory. The uniqueness of this platform is the enhancement of the NVLink interconnect by using NVSwitches [NVIDIA 2018c], which enable the connection of all 16 GPUs and higher bandwidth.

Similar machines can be found in the portfolio of many cloud providers and HPC centers. The key hardware parameters of both platforms related to this article are summarized in Table 1, for more details see Appendix B.

\subsection{Benchmark Scenes}

We used four scenes for benchmarking. The first one was the Moana Island Scene [Walt Disney Animation Studios 2018]. It was
Table 1. Parameters of HW Platforms Used for Validation of Our Proposed Approach

\begin{tabular}{lllll}
\hline Server & GPUs & $\begin{array}{l}\text { Local memory } \\
\text { bandwidth \& } \\
\text { latency }\end{array}$ & $\begin{array}{l}\text { Remote memory } \\
\text { bandwidth \& } \\
\text { latency }\end{array}$ & $\begin{array}{l}\text { Total GPU } \\
\text { memory }\end{array}$ \\
\hline Barbora & $4 \times \mathrm{V} 100$ & $740 \mathrm{~GB} / \mathrm{s} 4 \mu \mathrm{s}$ & $48 \mathrm{~GB} / \mathrm{s} 7 \mu \mathrm{s}$ & $64 \mathrm{~GB}$ \\
\hline DGX-2 & $16 \times \mathrm{V} 100$ & $790 \mathrm{~GB} / \mathrm{s} 4 \mu \mathrm{s}$ & $138 \mathrm{~GB} / \mathrm{s} 10 \mu \mathrm{s}$ & $512 \mathrm{~GB}$ \\
\hline
\end{tabular}

Bandwidth and latency is measured by the STREAM benchmark.

selected due to its uniqueness and wide acceptance by both industry and the research community as a key production grade benchmark. The second one is based on the Natural History Museum scene [Birn 2015], and is extended by sculpture models [Threedscans 2020] to increase the geometric complexity, and by paintings [The Art Institute of Chicago 2020] to increase the size and number of textures. Finally, the remaining two scenes come from the recent open movies produced by the animation studio of the Blender Institute, namely, Agent 327: Operation Barbershop [Studio 2020a] and Spring [Studio 2020b].

These scenes of different sizes were created through the surface subdivision functionality and by increasing the texture resolution.

\section{DATA DISTRIBUTED MULTI-GPU PATH TRACING}

In this section, we propose a method designed to minimize the negative impact of the remote memory accesses on an algorithmic level to maximize path tracing performance and scalability.

We propose two approaches for splitting data structures used by a path tracer. Each approach works with a different granularity of data placement control. We also evaluate how different data distributions affect overall performance.

In Section 4.1, we describe a less complex methodology that works with entire data structures. Then, in Section 4.2, we introduce an advanced methodology that works with data structures divided into chunks, and controls how these chunks are placed in the memory of individual GPUs.

The description of the methodology in this section is demonstrated using the Moana 12 GB and Moana 27 GB scenes for the Barbora and DGX-2 servers, respectively. The 12 or 27 GB value refers to the overall size of all data structures used by Cycles for path tracing. The scenes of these sizes were selected, because they fit into the memory of a single GPU of the respective platform, and we are therefore able to provide a full scalability evaluation from 1 to 4 , or from 1 to 16 GPUs.

\subsection{Basic Distribution of Entire Data Structures}

There are approximately 40 data structures in Cycles that describe the scene. Structures are accessed only for reading. Every data structure can be independently replicated or distributed across GPUs.

4.1.1 Memory Access Analysis. We define the order in which data structures are replicated as a ratio of the total memory accesses to a particular data structure over its size. To be able to analyze its behavior, Cycles was modified to count the number of accesses to each data structure. The analysis was done on the first sample when rendering the scenes with a resolution of $5,120 \times 2,560$ pixels. 
The structures that have the highest number of memory accesses per byte are shown in Figure 2. These are:

- small_structures-a set of data structures smaller than $16 \mathrm{MB}$ (the most important one is svm_nodes, which stores Shader Virtual Machine (SVM) data and codes),

- bvh_nodes-stores the BVH tree without its leaves (leaves are stored in a separate structure),

- prim_tri_verts-holds coordinates of all vertices in the scene,

- prim_tri_index-is a set of all triangles in the scene and it contains indices to the prim_tri_verts.

As an example, we shall examine the Moana 27 GB scene on the DGX-2 server. Evidently, the most important data structure is $b v h \_$nodes, because it is responsible for $79.6 \%$ of all memory accesses. This means that if it is replicated in the memory of all GPUs, the $79.6 \%$ of all memory accesses will be to the local memory. See the solid black line in Figure 2. The size of this structure is $7.2 \mathrm{~GB}$, which represents $26.5 \%$ of the entire scene size.

The remaining lines in Figure 2 show how the path-tracing performance is affected by the distribution and replication of these data structures. $0 \%$ scene replication means that all data structures are split into $2 \mathrm{MB}$ chunks and these are distributed in a round robin fashion among all GPUs. One can see that this naive distribution leads to an almost $3.7 \times$ longer rendering time (370\%) in cases all 16 GPUs are used. The performance penalty decreases when using a smaller number of GPUs. The path-tracing time for fully replicated scenes is used as a baseline for relative rendering time evaluation. The relative rendering time is decreased to only $149 \%$ by replicating small_structures and bvh_nodes on all 16 GPUs. If in addition to small_structures and bvh_nodes, prim_tri_index and prim_tri_verts are also replicated, then the relative rendering time is only $109 \%$ while $40.7 \%$ of the scene is replicated and the rest is distributed.

Consequently, the total memory allocation per GPU is $12.1 \mathrm{~GB}^{2}$ instead of 27.2 GB.

4.1.2 Performance and Scalability Evaluation. The scalability of the proposed approach is evaluated for four different cases:

(1) all data structures are replicated-this case serves as a baseline as it achieves the best performance and scalability,

(2) all data structures are evenly distributed,

(3) small structures and bvh_nodes are replicated while all other data structures are distributed,

(4) small structures, bvh_nodes, prim_tri_index, and prim_tri verts are replicated while all other data structures are distributed.

For case (2), two different forms of data distribution are evaluated: (a) continuous distribution: the structures are divided into large chunks of a size equal to the structure size over a number of GPUs, and each GPU owns one chunk, (b) round robin distribution: the distributed structure is divided into chunks of $2 \mathrm{MB},{ }^{3}$ which are distributed in a round robin fashion.

\footnotetext{
${ }^{2} 11.1$ GB replicated $+(16.1 \mathrm{~GB} / 16$ GPUs $)$ distributed $=12.1 \mathrm{~GB}$ per GPU.

${ }^{3}$ The $2 \mathrm{MB}$ chunk is the smallest possible size, which still provides good performance and is optimal for scenes of these sizes. Smaller chunks always yield worse performance for the SetReadMostly memory hint, which replicates the chunks over all GPUs.
}
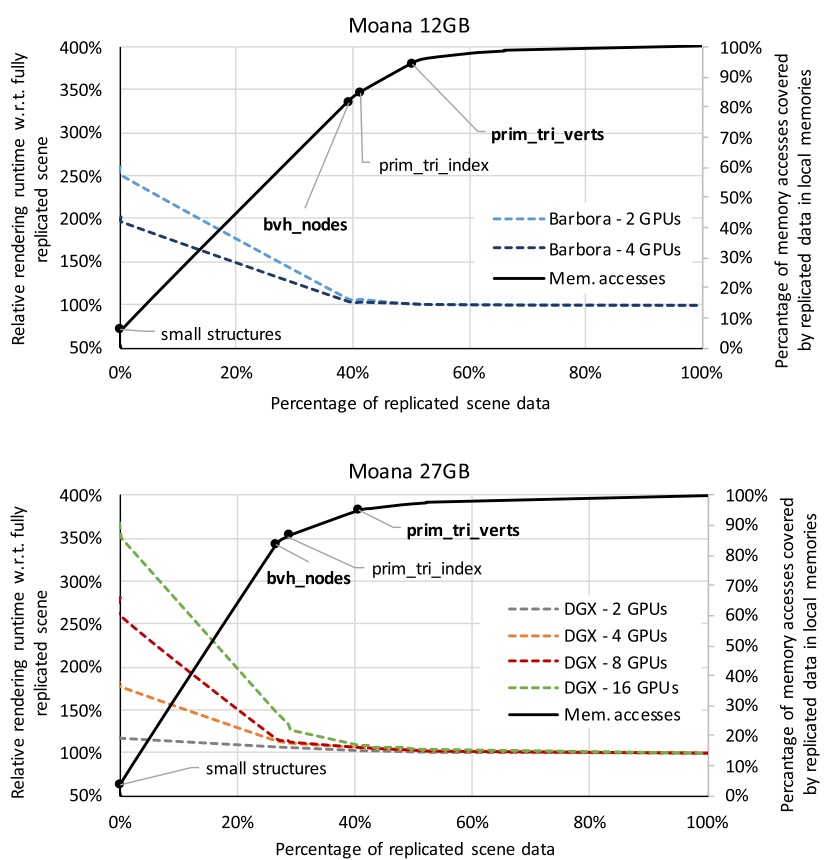

Fig. 2. Analysis of memory accesses covered by the most important data structures (solid black lines). Impact of the data structure distribution and replication on rendering performance. Results for $0 \%$ replication represent full distribution of all data structures.

The results of these tests are shown in Figure 3. The rendering times are for one sample per pixel and an image resolution of 5,120 $\times 2,560$ pixels. Several conclusions can be made from the presented results:

(1) round robin distribution of small chunks performs better than continuous distribution of large chunks, therefore it is always used to distribute non-replicated data structures,

(2) path tracing with fully distributed data structures does not scale on both platforms (there is reasonable scalability for two GPUs on DGX-2, but not beyond that),

(3) if small structures and $b v h \_n o d e s$ are replicated, the scalability is significantly improved:

- on Barbora the speedup is 3.8 for 4 GPUs,

- $\quad$ on DGX-2 the speedup is 7 and 6.7 for 8 GPUs and 11.8 and 11.5 for 16 GPUs for Moana 12GB and Moana 27GB scenes, respectively,

(4) if small structures, $b v h \_n o d e s$, prim_tri_index, and prim_tri_ verts are replicated, the scalability is further improved:

- on Barbora the speedup is 3.9 for 4 GPUs,

- on DGX-2 the speedup is 13.7 and 14.3 for 16 GPUs for Moana 12 GB and Moana 27 GB scenes, respectively,

\subsection{Advanced Distribution Based on Memory Access Pattern and Statistics}

Now, we present an advanced data placement algorithm that takes full advantage of the Unified Memory mechanism and data placement hints introduced in CUDA 8.0 (SetReadMostly, SetPreferredLocation, ...). 

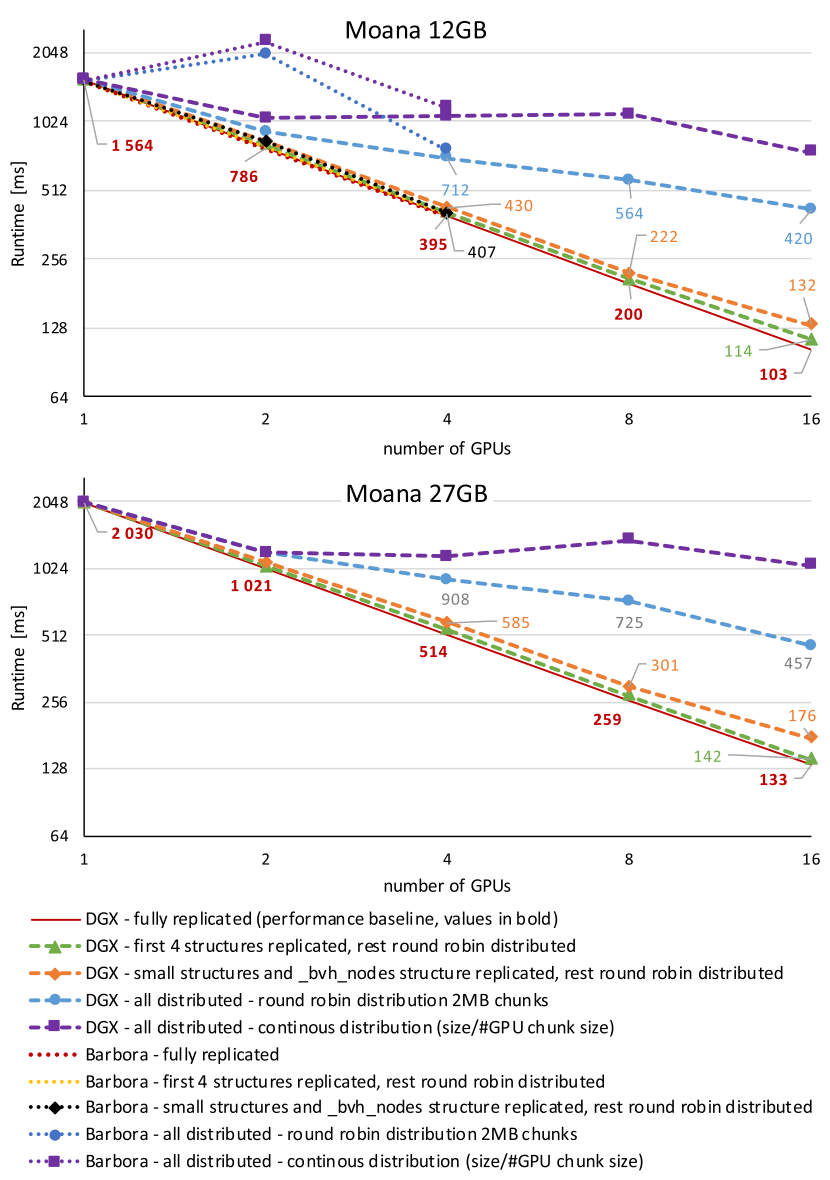

Fig. 3. Scalability of the Cycles path tracer for different data distribution using entire structures on the Barbora GPU server and DGX-2 for Moana 12 and 27 GB scenes. The rendering time is for one sample and the resolution is $5,120 \times 2,560$.

The data placement is done with chunks, and hints are set for each chunk individually. The optimal chunk size was identified experimentally by benchmarking the path tracer performance for chunks of sizes from $64 \mathrm{kB}$ to $128 \mathrm{MB}$. We observed that:

- for scenes smaller than $30 \mathrm{~GB}$ the optimal chunk size is $2 \mathrm{MB}$ (smaller chunks are not recommended),

- for scenes of sizes around 40 GB the optimal chunk size is $16 \mathrm{MB}$,

- for scenes of sizes above $120 \mathrm{~GB}$ the optimal chunk size is $64 \mathrm{MB}$.

The workflow of this data placement strategy can be summarized by the following steps (more details are given in the subsections below):

(1) copy/distribute every data structure across all GPUs in a round robin fashion using chunks of an optimal size,

(2) run the path tracing kernel with memory access counters for 1 spp to measure the statistics,

(3) gather the statistics on the CPU and run the proposed algorithm to get the optimal data chunks distribution,
(4) use cudaMemAdvise to migrate or replicate all chunks,

(5) run the original unmodified path tracing kernel.

4.2.1 Memory Access Pattern Analysis. To identify the memory access pattern, per chunk access counters have been implemented in the GPU path tracing kernel of Cycles. There are independent counters for all data structures and all their chunks, therefore, a total number of memory accesses per chunk can be recorded for each GPU.

The memory analysis starts with all data structures being evenly distributed using a round robin distribution. Then the modified path tracing kernel with memory access counters is executed on all GPUs for one sample.

When the kernel finishes, then for every chunk of every structure, a number of accesses from all GPUs is recorded. This data is the input for the data placement algorithm described in the next section. As is the case for final rendering, even during the analysis, the rendering workload is distributed, and each GPU works on its own part of the image. The workload is distributed among GPUs by horizontal stripes so that each GPU works on one stripe. Load balancing is done by changing the height of stripes.

The analysis of the memory accesses for the Moana scenes of different sizes is shown in Figure 4 . The figure shows that $1 \%$ of scene data covers between $56.7 \%$ and $74.4 \%$ of memory accesses, depending on the scene size. This is significantly better than working with entire structures. For illustration and direct comparison, we have added the dashed lines to Figure 4, which represent the solid black lines from Figure 2. Another important fact is that for the Moana 12 GB, 27 GB, 38 GB, and 169 GB scenes, 8.6\%, 17.1\%, $18.8 \%$, and $18.2 \%$, respectively, of the scene data chunks was not accessed at all.

Comparing these results with those in Figure 2, the following conclusions can be drawn:

- for the Moana 27 GB scene small_structures, bvh_nodes, prim_tri_index, and prim_tri_verts cover $95.1 \%$ of all memory access at cost of $40.7 \%$ scene replication-this results in $12.1 \mathrm{~GB}^{4}$ of data per GPU for 16 GPUs in total,

- for the same scene, the method presented in this section covers $95.1 \%$ of memory accesses with just $10.1 \%$ of replicated data-this results in $4.2 \mathrm{~GB}^{5}$ of data per GPU, which is 2.9 times smaller.

This analysis shows that there are clear candidates among the chunks that should be replicated on all GPUs, while a major portion of the data is accessed infrequently and can be distributed with an acceptable impact on performance. The algorithm that decides the placement of each chunk based on this analysis is described in the next section.

4.2.2 Data Placement Algorithm Based on Memory Access Pattern. Algorithm 1 processes the three-dimensional array of memory access counters Counters $[G][S][C]$, where $G$ are GPUs, $S$ are data structures, and $C$ are chunks within a particular data structure. The output of the algorithm provides an optimal location for each chunk (the GPU in which the chunk should be placed)

\footnotetext{
$\overline{{ }^{4} 11.1 \mathrm{~GB} \text { replicated }}+(16.1 \mathrm{~GB} / 16 \mathrm{GPUs})$ distributed $=12.1 \mathrm{~GB}$ per GPU. ${ }^{5} 2.7 \mathrm{~GB}$ replicated $+(24.5 \mathrm{~GB} / 16 \mathrm{GPUs})$ distributed $=4.2 \mathrm{~GB}$ per GPU.
} 


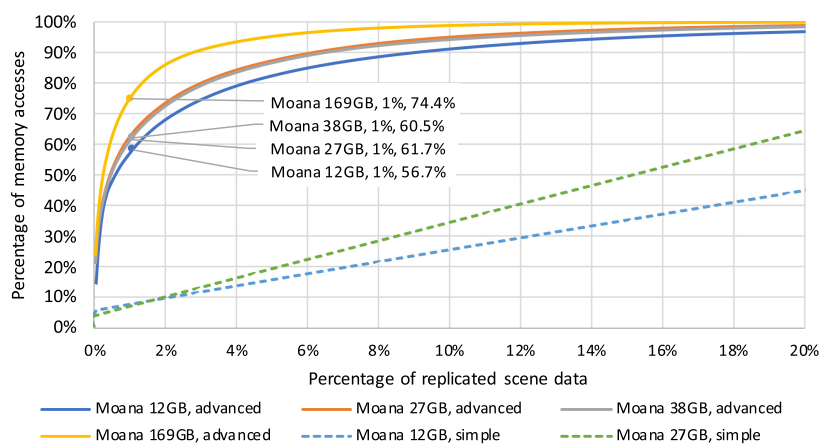

Fig. 4. The analysis of the memory accesses of the Cycles path tracer for Moana of 12, 27, 38, and 169 GB scenes. The figure shows that $1 \%$ of scene data broken into $2 \mathrm{MB}$ chunks covers between $57 \%$ and $74 \%$ memory accesses depending on scene size. For comparison, we have added the data points from Figure 2 as dashed lines to show the advantage of this method.

and decides whether the chunk should be distributed or replicated to all GPUs.

In the first step, the per GPU counters are summed to get the total number of accesses $a_{\text {sum }}$ for each chunk $c \in C$ of each data structure $s \in S$ and a $\left(a_{s u m}, s, c\right)$ tuple is created. One tuple now represent one chunk of scene data. Next, all tuples are put into a single $1 \mathrm{D}$ array $\mathrm{H}_{\text {comb }}$. The array is sorted by $a_{\text {sum }}$ from the largest value (the highest number of accesses) to the smallest one and stored in $\mathrm{H}_{<}$array.

The last input of the algorithm is the number of chunks that can be replicated $N_{\text {dup }}$. This value can either be set manually or automatically using the formula

$$
N_{\text {dup }}=\frac{1}{C_{s}}\left(G_{f}-\frac{S_{s}}{N_{g}}\right),
$$

where $G_{f}$ is the amount of free memory per GPU in MB available to store scene data, $S_{s}$ is the scene size in $\mathrm{MB}, N_{g}$ is the total number of GPUs, and $C_{s}$ is the chunk size in MB (2-64 MB based on scene size).

We define a threshold $t$ as the $N_{\text {dup }}$-th element in the sorted array $\mathrm{H}_{<}$and evaluate all tuples in the array $\mathrm{H}_{<}$. If the counter value $a_{\text {sum }}$ is larger than $t$, then the corresponding chunk will be set as SetReadMostly, and therefore replicated.

In the opposite case, the chunk is set as SetPreferredLocation and is assigned to the GPU with the highest number of accesses to this chunk. If the memory of this GPU is full, then the GPU with second, third, fourth, and so on, highest number of accesses is selected until a GPU with free memory is found.

If the counter value is equal to zero (without any accesses), then the corresponding chunk will be distributed in a round robin fashion across GPUs with free memory.

4.2.3 Performance Evaluation. The performance of the proposed algorithm was evaluated for different ratios between replicated and distributed data at a $2 \mathrm{MB}$ chunk level of granularity for the Moana 12 and 27 GB scenes. The range is from $0 \%$ of replicated chunks (fully distributed), all the way up to $100 \%$ of replicated chunks if possible. Please note that there is a difference in how chunks are distributed when compared to the approach presented in Section 4.1. Previously the chunks were placed in a naive
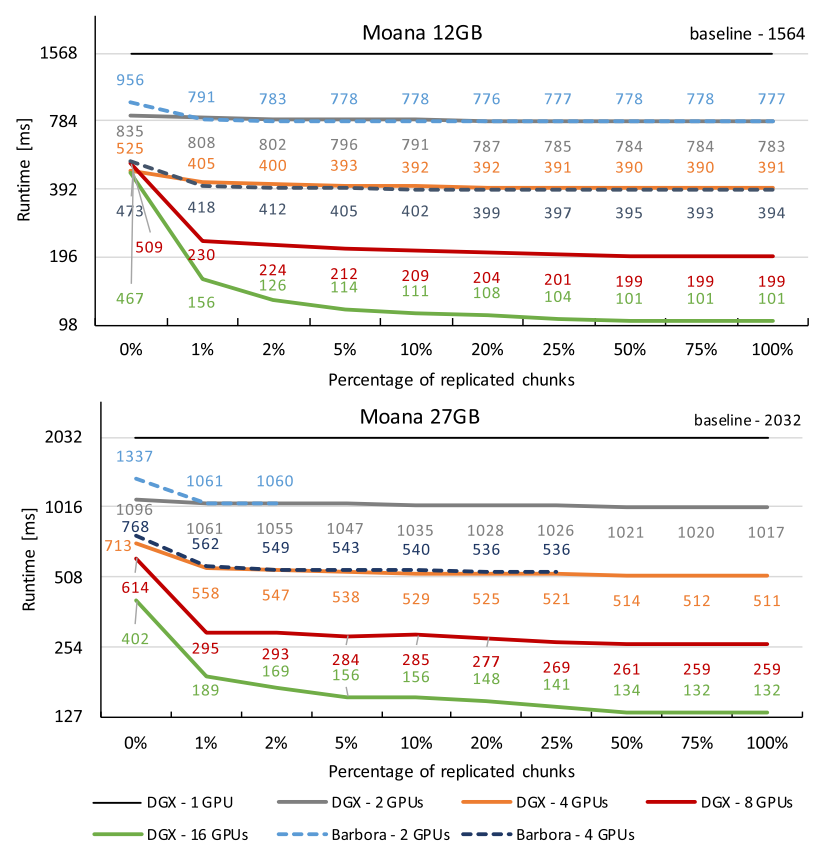

Fig. 5. Analysis of the path tracing performance for different ratios of replicated and distributed data for the Moana 12 GB and 27 GB scenes. Runtime is for one sample per pixel. A key observation is that the Barbora server with 4 GPUs with 16 GB of memory is able to render the Moana 27 $G B$ scene as fast as the DGX-2 system with 4 GPUs with 32 GB of memory in which the scene is fully replicated.

round robin fashion. However, the chunks are now placed in the memory of the GPU that had the highest number of accesses to it. Chunks are placed in the GPU memories so that each GPU has the same amount of data, whilst attempting to simultaneously place chunks into the memory of the GPUs that will access them the most. This explains the different and better performance for the fully distributed scenes, i.e., $0 \%$ replication.

Figure 5 shows the path tracing performance for one sample per pixel and 5, $120 \times 2,560$ pixel resolution for both scenes and platforms, and for all available GPUs. The baseline for evaluation is the runtime for $1 \mathrm{GPU}$ on DGX-2, which is 1,564 ms for the smaller scene and 2,032 ms for the larger scene. For the Moana 12 GB scene the speedup for fully a replicated scene on 4 GPUs on both platforms is 3.97, and parallel efficiency is $99 \%$. On DGX-2 the speedup for 16 GPUs is 15.5 , and the parallel efficiency is $97 \%$. For the Moana 27 GB scene and 16 GPUs the speedup is 15.4, and the parallel efficiency is $96 \%$. We expect these results to be positive as in this scenario the path tracing is an embarrassingly parallel problem.

When both platforms are compared, one can see that there is a performance difference only for fully distributed scenes. Once at least $1 \%$ of chunks are replicated, the performance is almost identical.

The Moana 27 GB scene does not fit into a single GPU memory used by the Barbora platform. For 2 GPUs, only up to $2 \%$ of chunks can be replicated. However, the parallel efficiency is still as high as $96 \%$ based on the single GPU baseline measured on the GPU in DGX-2 with 32 GB of memory. For 4 GPUs up to $25 \%$ of chunks can be replicated on the Barbora server, which yields 95\% parallel 


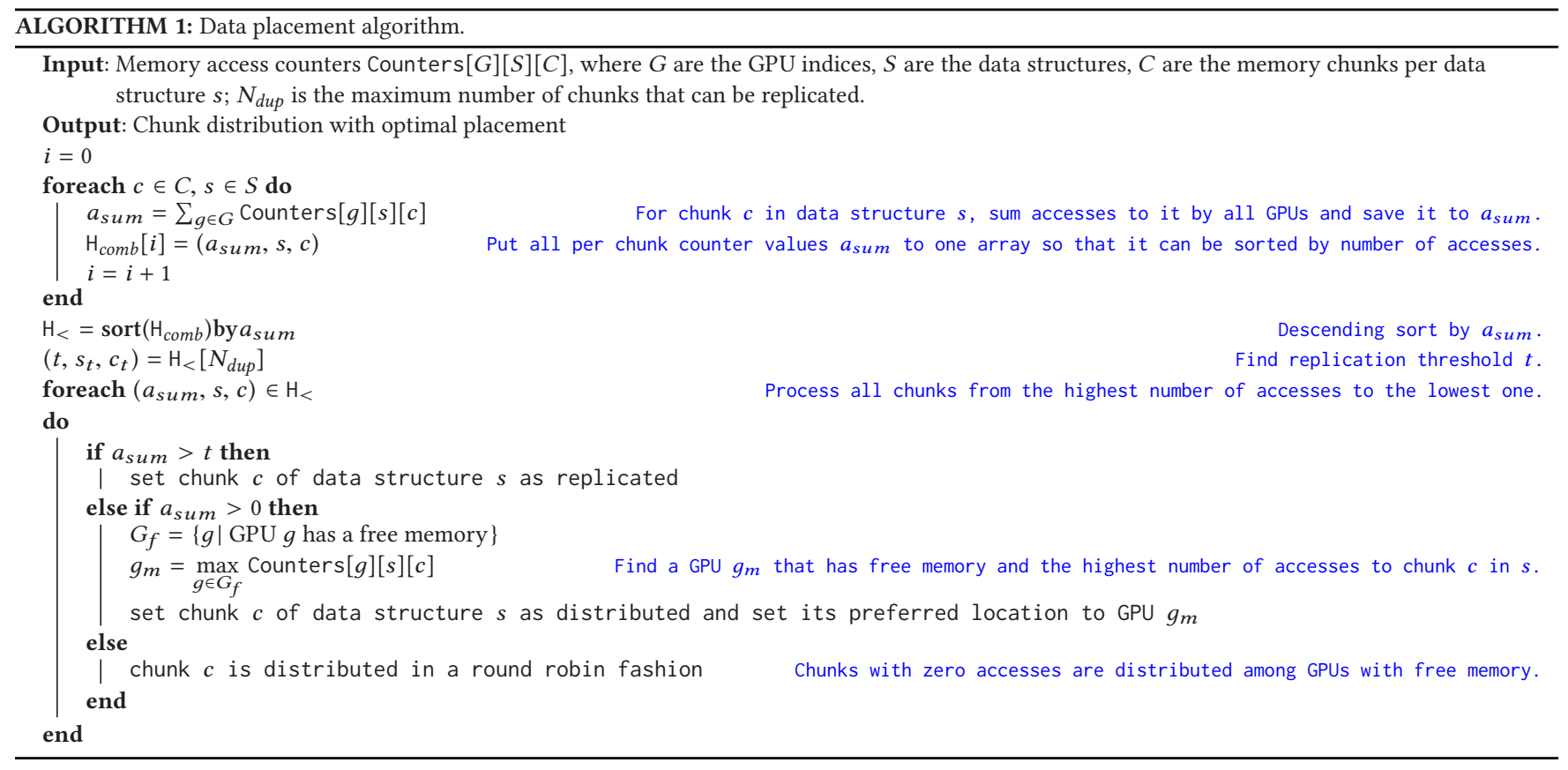

efficiency against the baseline. For comparison, if one uses 4 GPUs on DGX-2 with full scene replication, the parallel efficiency is $99 \%$. This is the first set of results that validates that path tracing on distributed scene data works efficiently.

If the same scene is processed on DGX-2 and 8 GPUs for $2 \%$, $10 \%, 20 \%$, and $25 \%$ scene replication, then the parallel performance is $86 \%, 89 \%, 92 \%$, and $94 \%$, respectively. The parallel efficiency for full scene replication is $98 \%$. For 16 GPUs the parallel efficiencies for the same scene replication ratios are $75 \%, 81 \%, 86 \%$, and $90 \%$. The baseline parallel efficiency is $96 \%$.

4.2.4 Maximum Scene Size Analysis. Up to this point, the scenes used for evaluation have had a size of 12 or 27 GB only. Based on the results from the previous section, the reader should now have an idea of how much of the scene data should be replicated to maintain performance.

The following equation describes the maximum ratio of replicated data that fits into the memory of GPU memory for a scene of a given size:

$$
N_{\text {max } \_d u p}=\frac{\left(G_{f}-\frac{S_{s}}{N_{g}}\right)}{\left(S_{s}-\frac{S_{s}}{N_{g}}\right)},
$$

where $G_{f}$ is the amount of free memory per GPU in MB available to store the scene data, $S_{s}$ is the scene size in $\mathrm{MB}$, and $N_{g}$ is the total number of GPUs.

For instance, the DGX-2 platform with 16 GPUs where each has $28 \mathrm{~GB}$ (out of $32 \mathrm{~GB}$ ) of memory available for the scene can render scenes of sizes of $256 \mathrm{~GB}, 179 \mathrm{~GB}, 138 \mathrm{~GB}$, and $112 \mathrm{~GB}$ that can have up to $5 \%, 10 \%, 15 \%$, and $20 \%$ of replicated data, respectively.

In the next section, one can see that larger scenes of around 100 GB need smaller percentages of replicated data than the smaller ones used here.

\section{PERFORMANCE FOR MASSIVE SCENES}

In this section, we evaluate the performance of the proposed method using two groups of scenes

(1) Group 1: Moana 38 GB, Museum 41 GB, Agent 37 GB, and Spring $41 G B$ are designed to stress the Barbora GPU server with 64 GB of total GPU memory, see Figure 6, and

(2) Group 2: Moana 169 GB, Museum 124 GB, Agent 167 GB, and Spring 137 GB are designed to stress the DGX-2 server with 512 GB of total GPU memory, see Figure $7 .^{6}$

All scenes can be seen in Figure 1 and the key parameters of the second group are shown in Table 3. All results are presented for one sample per pixel, but as Table 2 shows, these values can be linearly extrapolated to any number of samples per pixel. As the topic of out-of-core algorithms for textures is very extensively covered in the literature, we use scenes where both geometry and textures are larger than a single GPU memory and their ratio differs from scene to scene. The goal is to show that our approach is general, and works for textures as well as for geometry, which is the more challenging to distribute while maintaining good performance of path tracing.

The first key observation is that for different scene sizes, a different chunk size must be used to reach optimal performance, as presented in Section 4.2. For Group 1 the best performance was achieved with $16 \mathrm{MB}$ chunks. For Group 2 the optimal chunk size is $64 \mathrm{MB}$

The results for Group 1 are shown in Figure 6. The following conclusions can be made from the results:

\footnotetext{
${ }^{6}$ We were not able to work with larger scenes, because Cycles internally uses 32 bit integers for some key data structures.
} 

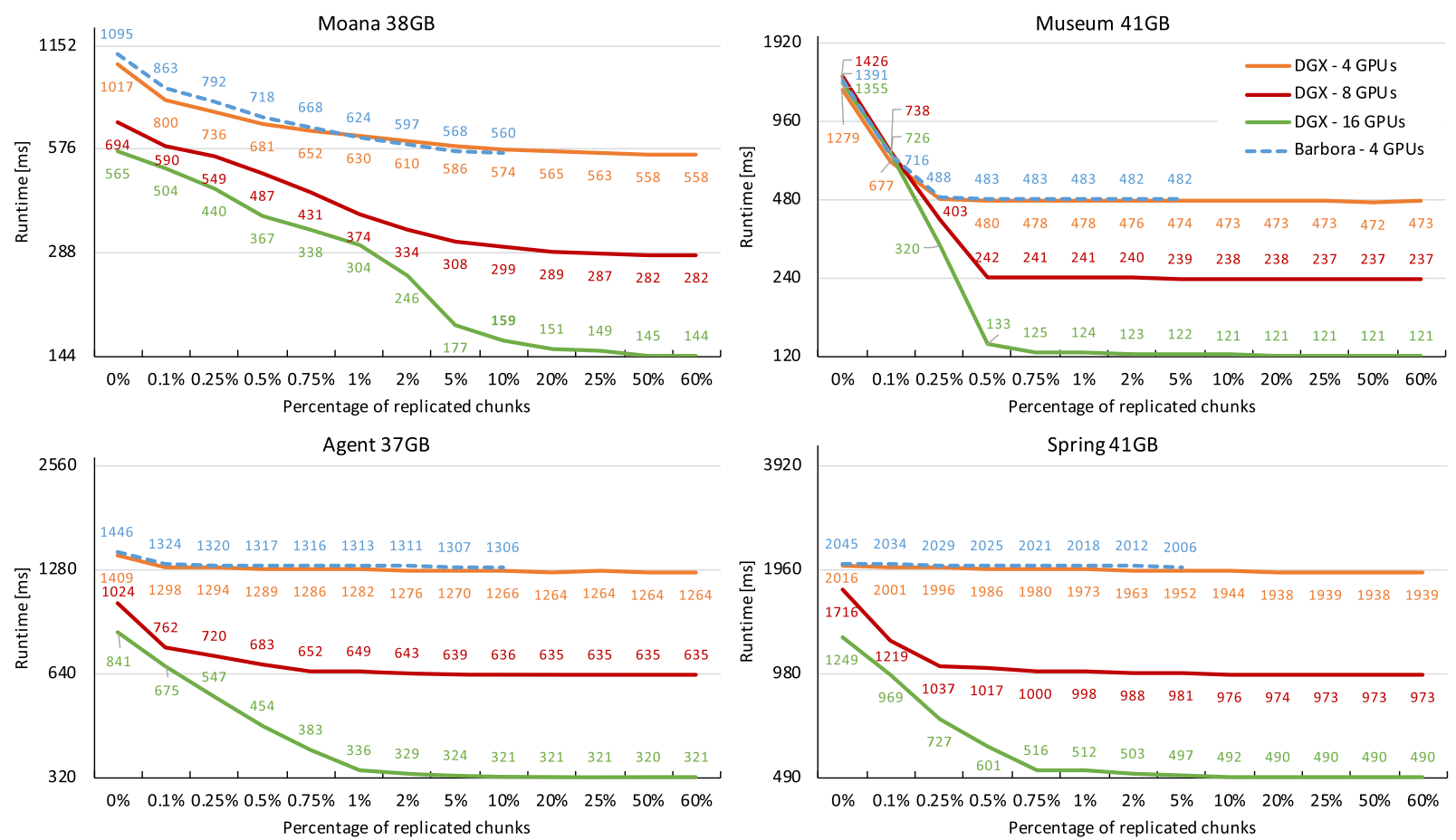

Fig. 6. Path tracing times for four large scenes (Group 1) running on 4 GPUs of the Barbora GPU server and 4, 8, and 16 GPUs of the DGX-2 system. The key observation is that the Barbora server with lower performing GPU interconnect has the same performance as the DGX-2 system for 4 GPUs.

Table 2. The Preprocessing Time and Final Rendering Time for the Different Number of Samples per Pixel Measured on 16 GPUs of the DGX-2 System

\begin{tabular}{lrrrr}
\hline Scene & Moana & Museum & $\begin{array}{r}\text { Agent } \\
\text { 169 GB }\end{array}$ & $\begin{array}{r}\text { Spring } \\
124 \mathrm{~GB}\end{array}$ \\
& & & & \\
\hline Preprocessing & & & & $137 \mathrm{~GB}$ \\
\hline 1spp pre-pass on GPUs & $0.75 \mathrm{~s}$ & $0.92 \mathrm{~s}$ & $0.74 \mathrm{~s}$ & $1.18 \mathrm{~s}$ \\
Algorithm runtime & $0.06 \mathrm{~s}$ & $0.01 \mathrm{~s}$ & $0.01 \mathrm{~s}$ & $0.01 \mathrm{~s}$ \\
Chunk redistribution & $66.1 \mathrm{~s}$ & $28.2 \mathrm{~s}$ & $50.9 \mathrm{~s}$ & $32.6 \mathrm{~s}$ \\
\hline Rendering & & & & \\
\hline 1 sample & $0.196 \mathrm{~s}$ & $0.125 \mathrm{~s}$ & $0.332 \mathrm{~s}$ & $0.498 \mathrm{~s}$ \\
10 samples & $1.94 \mathrm{~s}$ & $1.25 \mathrm{~s}$ & $3.37 \mathrm{~s}$ & $5.01 \mathrm{~s}$ \\
100 samples & $18.7 \mathrm{~s}$ & $12.6 \mathrm{~s}$ & $33.8 \mathrm{~s}$ & $50.1 \mathrm{~s}$ \\
1000 samples & $181 \mathrm{~s}$ & $126 \mathrm{~s}$ & $337 \mathrm{~s}$ & $502 \mathrm{~s}$ \\
10000 samples & $1852 \mathrm{~s}$ & $1309 \mathrm{~s}$ & $3378 \mathrm{~s}$ & $5016 \mathrm{~s}$ \\
\hline
\end{tabular}

Chunk size is $64 \mathrm{MB}$, replication ratio is $10 \%$. Access pattern and chunk distribution is calculated from first sample only, all other samples uses the same chunk distribution.

- the performance of the Barbora server is almost identical to the performance of DGX-2 for the same amount of scene replication (up to $10 \%$ ).

- DGX-2 is able to further replicate scene data up to $60 \%$, which improves performance by $2.8 \%$ only in the case of the Moana $38 \mathrm{~GB}$ scene (for the other scenes the performance is higher by only less than $1 \%^{7}$ ).

\footnotetext{
${ }^{7}$ Museum 41 GB by $0.2 \%$, Agent 37 GB by $0,2 \%$, and Spring 41 GB by $0.7 \%$.
}

- This means that for scenes of sizes approximately up to $45 \mathrm{~GB}$ distributed over 4 GPUs, the significantly less complex and cheaper GPU interconnect in the Barbora server is sufficient.

- For the Museum, Agent, and Spring scenes 2\% of scene replication attains optimal performance. This holds for 4,8 , and 16 GPUs.

- Only the Moana scene needs higher amounts of replicated data, up to $25 \%$ for 16 GPUs.

- Scalability can be evaluated on DGX-2 for 4, 8, and 16 GPUs only. For the Moana, Museum, Agent, and Spring scenes, for $5 \%$ scene replication, the parallel efficiencies, going from 4 to 16 GPUs, are $82.7 \%, 97.1 \%, 97.9 \%$, and $98.1 \%$, respectively. In the case of the Moana scene, a higher replication ratio is needed to improve scalability, e.g., for $25 \%$ data replication ratio the parallel efficiency is $94.4 \%$.

The results for Group 2 are shown in Figure 7. One can see that:

- The performance is affected by selecting the right chunk size, particularly for the Moana scene.

- For 8 GPUs and the Moana, Agent, and Spring scenes, if the replication ratio is $10 \%$ the scene does not fit into GPU shared memory anymore and chunks are swapped between GPU and CPU memory, ${ }^{8}$ which makes the rendering several times slower depending on the number of chunks being moved back and forth. This is the point at which our approach stops working, and therefore it is crucial to correctly select the replication ratio to avoid this situation.

\footnotetext{
${ }^{8}$ This is the default behavior of the CUDA Unified Memory.
} 

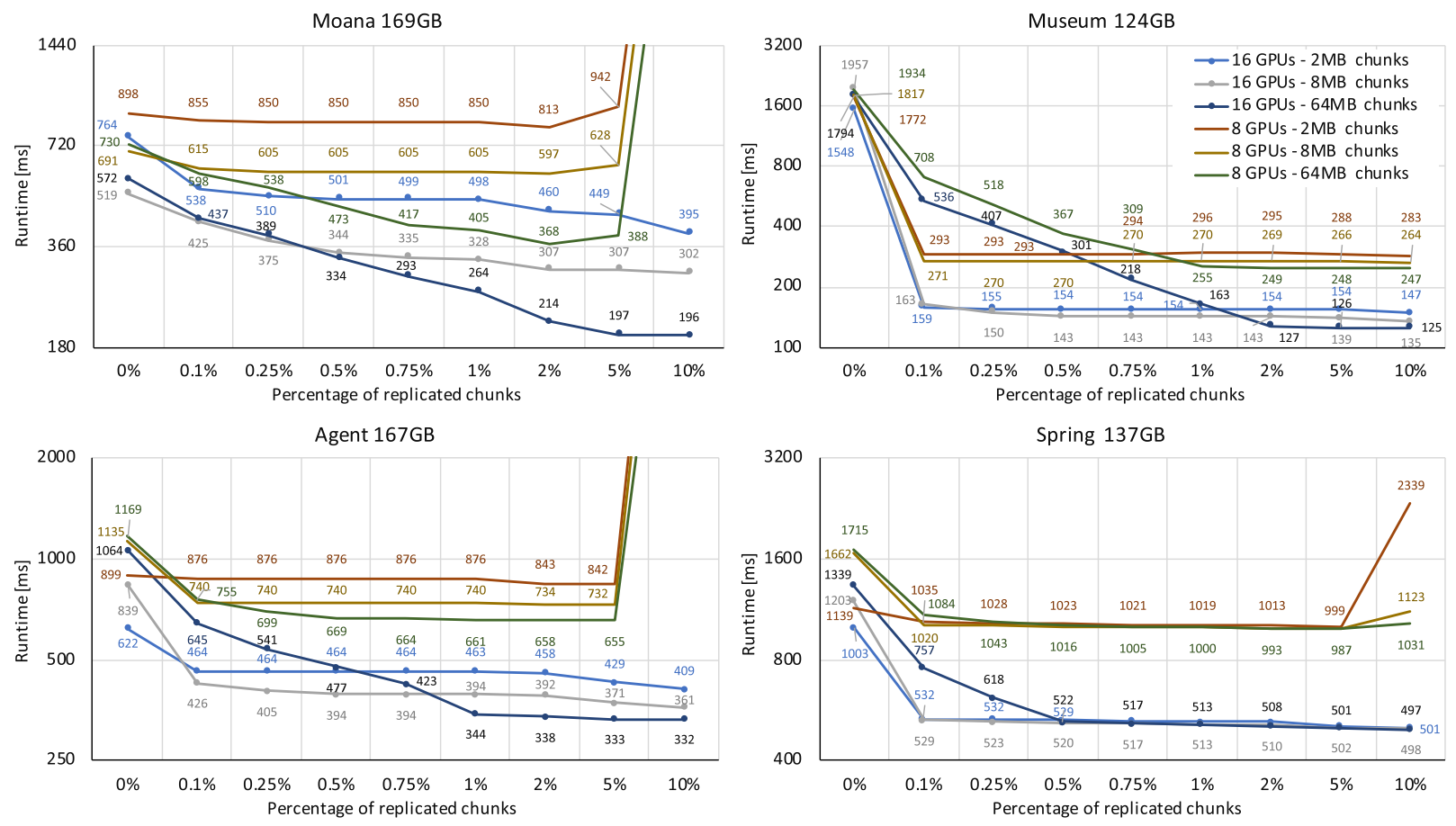

Fig. 7. Path tracing times for the four largest scenes (Group 2) running on 8 and 16 GPUs of the DGX-2 system. The results show how the performance is affected by changing the chunk size from 8 to $64 \mathrm{~GB}$. The runtimes are for one sample per pixel. $0 \%$ of replicated chunks represents the fully distributed scene.

- For the Agent scene $1 \%$ of scene replication gives optimal performance for both 8 and 16 GPUs. The Spring scenes needs only $0.1 \%$ for 8 GPUs and $0.25 \%$ for 16 GPUs. The Museum scene needs $1 \%$ for 8 GPUs and $2 \%$ for 16 GPUs. Finally, the Moana scene requires $2 \%$ for 8 GPUs and $5 \%$ for 16 GPUs.

- Scalability between 8 and 16 GPUs is good for all scenes. The parallel efficiencies are $93.8 \%, 98.8 \%, 98.6 \%$, and $99.0 \%$ for the Moana, Museum, Agent, and Spring scenes, respectively.

Another important feature of the proposed approach is that we analyze memory access pattern only for one sample per pixel. Based on this pattern, we distribute and replicate the chunks only once, and then use this chunk placement for all remaining samples. The cost of this preprocessing is shown in Table 2. We have verified that it is sufficient through a set of measurements using Group2 scenes for 1 to 10,000 samples. The results are in the same table, where one can see that rendering times grows linearly with the number of samples.

Finally, for Group 2 scenes, we have evaluated Cycles performance on 16 GPUs for different path tracer settings, see columns 1-3 in Table 4. The table also shows the recorded total number of bounces for all scenes and the four settings. Please note that all the remaining measurements in this article were done using the default settings. The results of this experiment are shown in Figure 8. One can see that our approach works for all four settings of the path tracer and the performance is only proportionally affected by the total number of bounces. For instance, in case of the Museum 124 GB scene, by changing the settings from default to full, the total number of bounces increases 1.7 times and rendering time increases 2.9 times (from $125 \mathrm{~ms}$ to $360 \mathrm{~ms}$ ).

\section{CONCLUSIONS}

In this article, we have presented a solution for path tracing of massive scenes on multiple GPUs. Our approach analyzes the memory access pattern of a path tracer and defines how the scene data should be distributed across GPUs with a minimal loss of performance. The key concept is that those parts of the scene data that have the highest memory access rate are replicated on all GPUs, because their distribution would have a major negative impact on performance.

We propose two methods. They both work at the memory management level but with different granularity. Working at the memory management level means that one only controls where (what GPU) and how (replicate or distribute) the data structures are allocated, and that one does not care about what is stored in the data structure. By different granularity, we mean that: (i) the first approach uses the same memory management rules for the entire data structure; (ii) the second approach splits the data structures into chunks and we control the placement and/or replication of each chunk separately. Since we only control the memory allocations, the path tracer data structures do not have to be redesigned. This makes our approach applicable to other GPUbased path tracers with minor changes in their code. As a proofof-concept, we have enhanced the popular open-source Blender Cycles path tracer, which has already been successfully used in many open-source and commercial projects.

An important element of our approach is also the hardware. We take full advantage of NVLink 2.0 interconnect and its high bandwidth and low latency. We verified the method on two systems: (i) the more affordable 4 GPU server Barbora with lower 
Table 3. Parameters of the Largest Scenes Used for Performance Evaluation of the Presented Approach on DGX-2

\begin{tabular}{|c|c|c|c|c|}
\hline Scene & Moana 169GB & Museum 124GB & Agent 167GB & Spring 137GB \\
\hline GPU memory needed for path tracing & $169 \mathrm{~GB}$ & $124 \mathrm{~GB}$ & $167 \mathrm{~GB}$ & $137 \mathrm{~GB}$ \\
\hline Image resolution & $5,120 \times 2,560$ & $5,120 \times 2,560$ & $5,120 \times 2,560$ & $5,120 \times 2,560$ \\
\hline Geometry size & $90 \mathrm{~GB}$ & $69 \mathrm{~GB}$ & $57 \mathrm{~GB}$ & $48 \mathrm{~GB}$ \\
\hline Triangles count & $673 \mathrm{M}$ & $610 \mathrm{M}$ & $468 \mathrm{M}$ & $395 \mathrm{M}$ \\
\hline Total textures size & $58 \mathrm{~GB}$ & $46 \mathrm{~GB}$ & $101 \mathrm{~GB}$ & $74 \mathrm{~GB}$ \\
\hline Number of textures & 3421 & 22 & 118 & 96 \\
\hline \multicolumn{5}{|c|}{ Sizes of key data structures and percentage of total scene size } \\
\hline bvh_nodes & $14 \mathrm{~GB}(8 \%)$ & $8 \mathrm{~GB}(6 \%)$ & $6 \mathrm{~GB}(4 \%)$ & $7 \mathrm{~GB}(5 \%)$ \\
\hline prim_tri_verts & $30 \mathrm{~GB}(18 \%)$ & $27 \mathrm{~GB}(22 \%)$ & $21 \mathrm{~GB}(13 \%)$ & $18 \mathrm{~GB}(13 \%)$ \\
\hline prim_tri_index & 3 GB $(2 \%)$ & $2 \mathrm{~GB}(2 \%)$ & $2 \mathrm{~GB}(1 \%)$ & 2 GB $(1 \%)$ \\
\hline svm_nodes & $2 \mathrm{MB}(<0.1 \%)$ & $40 \mathrm{kB}(<0.1 \%)$ & $4 \mathrm{MB}(<0.1 \%)$ & $5 \mathrm{MB}(<0.1 \%)$ \\
\hline tex_image & $58 \mathrm{~GB}(34 \%)$ & $46 \mathrm{~GB}(37 \%)$ & $101 \mathrm{~GB}(61 \%)$ & $74 \mathrm{~GB}(54 \%)$ \\
\hline \multicolumn{5}{|c|}{ Percentage of total memory access for key data structures for default bounces } \\
\hline bvh_nodes & $80.3 \%$ & $66.8 \%$ & $52.8 \%$ & $62.8 \%$ \\
\hline prim_tri_verts & $8.1 \%$ & $12.7 \%$ & $8.7 \%$ & $4.8 \%$ \\
\hline prim_tri_index & $2.6 \%$ & $3.9 \%$ & $2.6 \%$ & $1.5 \%$ \\
\hline svm_nodes & $2.1 \%$ & $3.8 \%$ & $19.3 \%$ & $19.2 \%$ \\
\hline tex_image & $0.2 \%$ & $0.5 \%$ & $3.4 \%$ & $2.3 \%$ \\
\hline
\end{tabular}

Average, across all GPUs, amount of replication per data structure for $64 \mathrm{MB}$ chunks and 16 GPUs. The values are for

$0,1,2,5$, and $10 \%$ of replicated chunks. For example, the bold value means that each GPU keeps on average $28 \%$ of the bvh_nodes in its local memory if $2 \%$ of the scene is replicated.

\begin{tabular}{lllll}
\hline bvh_nodes $^{9}$ & $6,17, \mathbf{2 8}, 50,62 \%$ & $6,16,26,61,88 \%$ & $6,25,33,44,64 \%$ & $6,20,25,31,36 \%$ \\
prim_tri_verts $^{10}$ & $6,6,6,10,20 \%$ & $6,7,7,7,16 \%$ & $6,7,10,14,20 \%$ & $6,7,10,16,23 \%$ \\
prim_tri_index $^{11}$ & $6,6,6,27,48 \%$ & $6,11,11,19,57 \%$ & $6,16,19,39,52 \%$ & $6,10,17,39,46 \%$ \\
svm_nodes $^{12}$ & $6,100,100,100,100 \%$ & $6,100,100,100,100 \%$ & $6,100,100,100,100 \%$ & $6,100,100,100,100 \%$ \\
tex_image $^{13}$ & $6,6,6,6,6 \%$ & $6,6,7,7,7 \%$ & $6,6,6,7,10 \%$ & $6,6,6,7,11 \%$ \\
\hline
\end{tabular}

performing interconnect $(3 \times$ lower bandwidth between GPUs, but $1.4 \times$ lower latency when compared to DGX-2) and 16GB of memory per GPU, and (ii) the DGX-2 server with 16 GPUs (each with 32 GB of memory) and state-of-the-art GPU interconnect with inter-GPU bandwidth comparable to the local memory bandwidth of a high-end CPU.

We used the Moana Island, Museum, Agent, and Spring scenes of two sizes each for benchmarking and validation. The first group of scenes is approximately $40 \mathrm{~GB}$ and is used primarily to validate the approach on the Barbora server. Our results prove that for such scenes its configuration is sufficient and path tracer takes full advantage of all 4 GPUs, as can be seen in Figure 6. On the DGX-2 system, the Museum, Agent, and Spring scenes needs only $2 \%$ of scene replication to reach optimal performance up to 16 GPUs. In terms of scalability the parallel efficiency for these three scenes is above $97 \%$, going from 4 to 16 GPUs. The Moana scene needs higher replication, up to $25 \%$ for 16 GPUs. For this configuration the parallel efficiency is $94 \%$.

The second group of scenes is approximately $150 \mathrm{~GB}$ and is designed for DGX-2. For these large scenes $1-2 \%$ of replication is

\footnotetext{
${ }^{9}$ Stores BVH tree without its leaves.

${ }^{10}$ Stores coordinates of all vertices in the scene.

${ }^{11}$ Stores all objects of type triangle.

${ }^{12}$ Stores Shared Virtual Machine data and codes.

${ }^{13}$ Stores all textures.
}

sufficient for near optimal performance. Only the Moana scene requires $5 \%$ replication for 16 GPUs. Scalability from 8 to 16 GPUs is good for all scenes as parallel efficiencies are above $98 \%$. Only for the Moana scene it is $94 \%$.

The data replication is proposed as the essential technique to maintain the near optimal performance of the path tracer. Nevertheless due to replication one cannot render scenes as large as the GPU shared memory. We show that only $1-5 \%$ of a scene needs to be replicated for scenes of a size over $124 \mathrm{~GB}$. For this range of replication, the DGX-2 with $512 \mathrm{~GB}$ of memory can render scenes of sizes between 250 and 390 GB.

We believe that our approach can boost the adaptation of multiGPU rendering by industry where path tracing of large scenes is essential. To the best of our knowledge, this article presents the first work dealing with efficient utilization of more than two GPUs for path tracing of massive scenes where scene data including geometry is distributed.

\section{FUTURE WORK}

We plan to extend our work to support POWER9 platforms accelerated by multiple Tesla V100s. The key reason is that each POWER9 CPU is equipped with NVLink 2.0. With such platforms, our approach can be enhanced by an efficient out-of-core mechanism that uses CPU memory for rarely accessed data. 

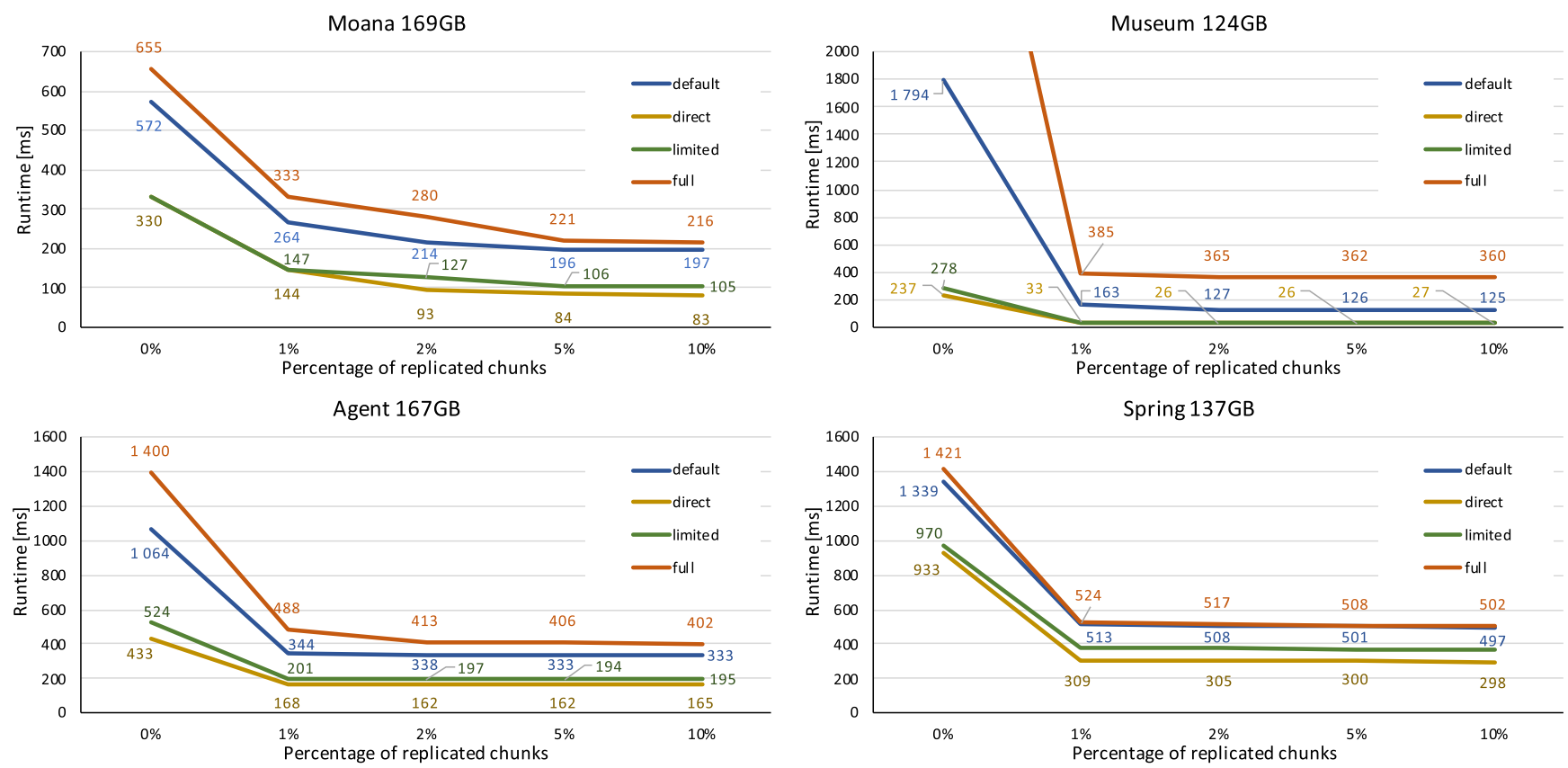

Fig. 8. Path tracing times for the four largest scenes (Group 2) running on 16 GPUs using a different path-tracing setup. The runtimes are for one sample per pixel.

Table 4. Total Number of Bounces for Different Setting Profiles of Cycles Path Tracer

\begin{tabular}{|c|c|c|c|c|c|c|}
\hline & \multicolumn{2}{|c|}{ Path tracer settings } & \multicolumn{4}{|c|}{ Measured number of bounces for all pixels and one sample per pix. } \\
\hline Profile name & $\begin{array}{l}\text { Bounce } \\
\text { type }\end{array}$ & $\begin{array}{l}\text { Max. \# of bounces } \\
\text { per sample }\end{array}$ & Moana 169 GB & Museum $124 \mathrm{~GB}$ & Agent $167 \mathrm{~GB}$ & Spring $137 \mathrm{~GB}$ \\
\hline \multirow[t]{5}{*}{ Direct } & Total & 8 & $10,541 \mathrm{k}$ & $12,938 \mathrm{k}$ & $12,617 \mathrm{k}$ & $12,727 \mathrm{k}$ \\
\hline & Diffuse & 0 & $7,768 \mathrm{k}$ & $11,211 \mathrm{k}$ & $10,256 \mathrm{k}$ & $9,392 \mathrm{k}$ \\
\hline & Glossy & 1 & $1,780 \mathrm{k}$ & $1,727 \mathrm{k}$ & $1,906 \mathrm{k}$ & $2,976 \mathrm{k}$ \\
\hline & Transparency & 8 & 0 & 64 & $950 \mathrm{k}$ & $42 \mathrm{k}$ \\
\hline & Transmission & 2 & $993 \mathrm{k}$ & 0 & $454 \mathrm{k}$ & $359 \mathrm{k}$ \\
\hline \multirow[t]{5}{*}{ Limited } & Total & 8 & $12,006 \mathrm{k}$ & $13,963 \mathrm{k}$ & $13,783 \mathrm{k}$ & $13,771 \mathrm{k}$ \\
\hline & Diffuse & 1 & $9,142 \mathrm{k}$ & $12,101 \mathrm{k}$ & $11,171 \mathrm{k}$ & $10,129 \mathrm{k}$ \\
\hline & Glossy & 4 & $1,859 \mathrm{k}$ & $1,835 \mathrm{k}$ & $2,047 \mathrm{k}$ & $3,232 \mathrm{k}$ \\
\hline & Transparency & 8 & 0 & 63 & $901 \mathrm{k}$ & $42 \mathrm{k}$ \\
\hline & Transmission & 8 & $1,005 \mathrm{k}$ & $27 \mathrm{k}$ & $565 \mathrm{k}$ & $409 \mathrm{k}$ \\
\hline \multirow[t]{5}{*}{ Default } & Total & 12 & $20,641 \mathrm{k}$ & $40,578 \mathrm{k}$ & $22,583 \mathrm{k}$ & $18,144 \mathrm{k}$ \\
\hline & Diffuse & 4 & $16,458 \mathrm{k}$ & $35,475 \mathrm{k}$ & $19,934 \mathrm{k}$ & $14,391 \mathrm{k}$ \\
\hline & Glossy & 4 & $2,465 \mathrm{k}$ & $3,920 \mathrm{k}$ & $2,052 \mathrm{k}$ & $3,233 \mathrm{k}$ \\
\hline & Transparency & 8 & 0 & 61 & $936 \mathrm{k}$ & $40 \mathrm{k}$ \\
\hline & Transmission & 12 & $1,718 \mathrm{k}$ & $1,184 \mathrm{k}$ & $597 \mathrm{k}$ & $520 \mathrm{k}$ \\
\hline \multirow[t]{5}{*}{ Full } & Total & 128 & $22,040 \mathrm{k}$ & $67,788 \mathrm{k}$ & $23,580 \mathrm{k}$ & $18,302 \mathrm{k}$ \\
\hline & Diffuse & 128 & $17,800 \mathrm{k}$ & $60,052 \mathrm{k}$ & $20,928 \mathrm{k}$ & $14,537 \mathrm{k}$ \\
\hline & Glossy & 128 & $2,493 \mathrm{k}$ & $5,573 \mathrm{k}$ & $2,052 \mathrm{k}$ & $3,234 \mathrm{k}$ \\
\hline & Transparency & 128 & 0 & 69 & $941 \mathrm{k}$ & $40 \mathrm{k}$ \\
\hline & Transmission & 128 & $1,747 \mathrm{k}$ & $2,163 \mathrm{k}$ & $599 \mathrm{k}$ & $531 \mathrm{k}$ \\
\hline
\end{tabular}

\section{APPENDICES}

\section{A IMPORTING THE MOANA ISLAND SCENE INTO BLENDER}

Several actions had to be taken to load the Moana Island scene [Walt Disney Animation Studios 2018] into Blender. The geome- try is imported in one unifying Blender object. To each individual geometry that is imported it is assigned its own empty object in the scene. The empty objects are created using the Particles/Hair modifiers. In this way the object instantiation is solved.

For surface subdivision, Blender offers an implementation that uses OpenSUBDIV from Pixar and Dreamworks [PIXAR 2019]. 


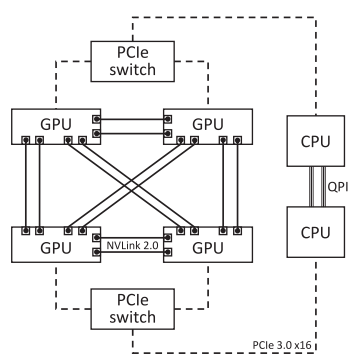

Fig. 9. NVLink GPU interconnect in the Barbora GPU server.

Concerning the materials, Blender provides Principled BSDF that is analogous to Disney's Principled BRDF/BSDF. The textures are loaded using the PTEX library from Pixar [Burley and Lacewell 2008] and converted to the OpenEXR [Kainz et al. 2009] format with UV mapping, which is supported by Blender. The result of this work is a new Blender add-on.

\section{B PATH-TRACING HARDWARE DESCRIPTION}

\section{B.1 Barbora GPU Server Hardware Description}

The BullSequana X410 E5 is a dense GPU-accelerated compute node developed for HPC infrastructures, which contains two CPUs and 4 Tesla V100 GPUs, each with 16 GB of fast HBM2 memory. For the purpose of this article the key part is the NVLink 2.0 [Sarosh 2019] interconnect that connects all GPUs as shown in Figure 9. Tesla V100 contains 6 NVlink 2.0 ports, each capable of a theoretical bandwidth of $25 \mathrm{~GB} / \mathrm{s}$ in each direction. Each GPU is connected to 3 other GPUs and there are two links between any pair of GPUs. This provides a total bandwidth of $50 \mathrm{~GB} / \mathrm{s}$. This is the theoretical bandwidth at which one GPU can read/write data from/to another GPU memory.

The actual remote memory bandwidth measured by the STREAM benchmark [McCalpin 1995] is $48 \mathrm{~GB} / \mathrm{s}$ for all combinations of GPUs, as shown in Figure 12. The latency is approximately $7 \mu \mathrm{s}$. The local memory bandwidth is $743 \mathrm{~GB} / \mathrm{s}$, which is 15.5 times higher than the remote memory bandwidth. The article shows that even though the remote memory bandwidth is 2.9 times lower than in the case of DGX-2 (see next section), it is still sufficient for our approach if path-tracing uses four 4 GPUs only.

\section{B.2 Nvidia DGX-2 Hardware Description}

There are multiple platforms on the market that are equipped with multiple GPUs connected using NVLink [Atos 2017; Sarosh 2019]. However, currently only NVIDIA HGX-2 and DGX-2 platforms use NVSwitch [NVIDIA 2018c]-based interconnect to connect 16 Tesla V100 GPUs. The theoretical bandwidth between any pair of GPUs is $150 \mathrm{~GB} / \mathrm{s}$ in one direction (bidirectional bandwidth is $300 \mathrm{~GB} / \mathrm{s}) .{ }^{14}$ Because of its advanced interconnect, any set of 8 GPUs can communicate with the remaining 8 GPUs in parallel, and any pair of GPUs communicate at the full $300 \mathrm{~GB} / \mathrm{s}$ bandwidth. This is facilitated by the non-blocking Fat tree topology of the NVSwitch network; see Figure 10. This unique hardware feature is also an essential one for the approach presented in this ar-

${ }^{14}$ This bandwidth is achieved by combining all 6 NVLink 2.0 links available at the Tesla V100.

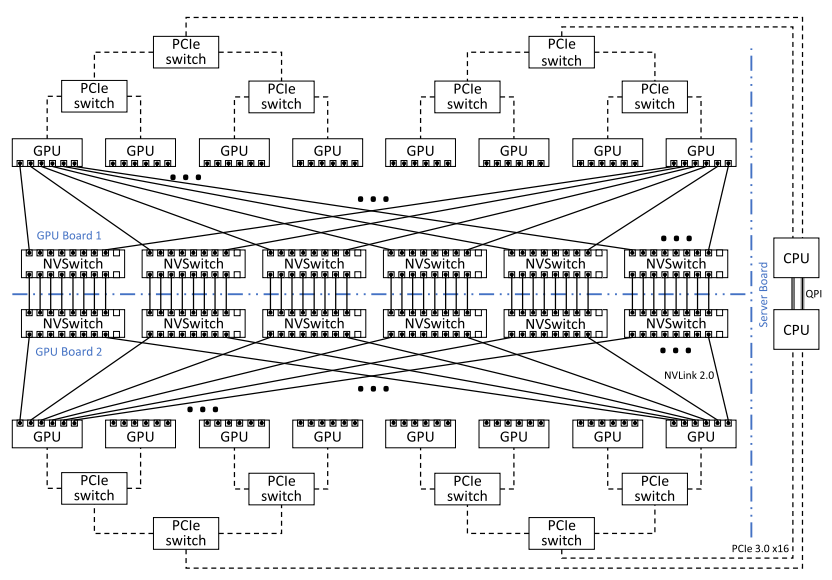

Fig. 10. NVlink GPU interconnect in the DGX-2 system.

ticle, because: (i) it enables fast access for a GPU to the memory of another GPU, and (ii) with UM technology (see Appendix C for more details) it gives a code developer 512 GB of global GPU shared memory.

Real memory bandwidth and latency to local and remote memory was measured using a specialized benchmark [Li et al. 2018] for both NVLink 2.0 and PCI-Express $3.0^{15}$ buses. The results for NVLink are shown in Figures 11(b) and 11(d) and the results for PCI-Express are shown in Figures 11(a) and 11(c). The values on the diagonal represent performance of the local memory, and off-diagonal values represent access to remote memory. One can see that local memory has approximately 5.7 times higher bandwidth and 3 times lower latency when compared to reading remote memory over NVLink. Also the bandwidth of NVLink is 12.5 times higher than the bandwidth of PCI-Express, and the latency is 2.5 times lower. When compared to state-of-the-art CPUs (with 8 memory channels) with a memory bandwidth, which, when measured by the STREAM benchmark [McCalpin 1995], is approximately $147 \mathrm{~GB} / \mathrm{s}$, one can see that this bandwidth is only $6 \%$ higher than the real NVLink bandwidth [AMD 2017].

These values suggest that the technology has reached the point where data distributed GPU path tracing becomes possible. However, the hardware is not a panacea. It is still necessary to minimize the negative impact of remote memory access as much as possible to achieve good path tracing performance and scalability, as shown in Section 4.

\section{B.3 Performance of Tesla V100 for Path Tracing in Blender Cycles}

In this section, we present a performance comparison of a Tesla V100 GPU with the top-of-the-line GeForce RTX 2080 Ti consumer grade card, which is based on Turing architecture and therefore equipped with hardware acceleration for ray tracing using RT cores. The main goal is to provide information about the path tracing performance of Tesla V100 against a well-known and widely used GPU card. The results are shown in Figure 14. Please focus on the comparison of Cycles with OptiX7.0@GeForce RTX 2080 Ti,

\footnotetext{
${ }^{15}$ Using 16 PCI-Express lanes.
} 


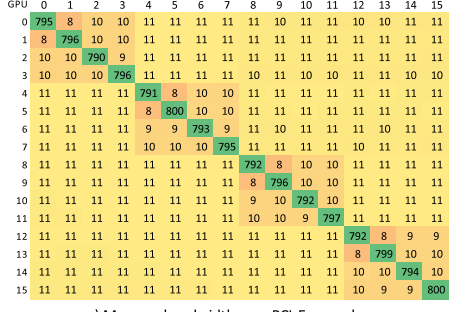

a.) Memory bandwidth over PCl-Express bus

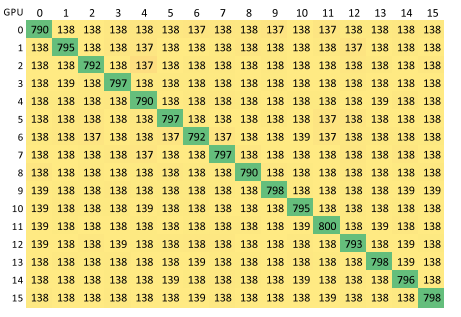

b.) Memory bandwidth over NVLink bus

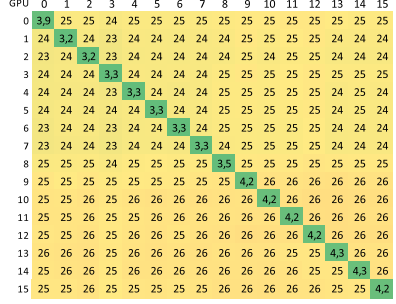

c.) Memory access latency over PCl-Express bus

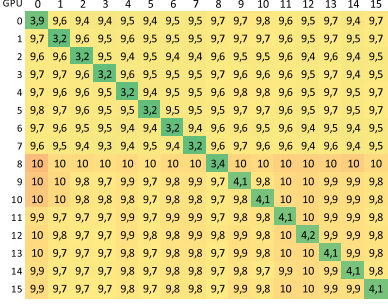

d.) Memory access latency over NVLink

Fig. 11. Comparison of bandwidth and latency for accessing remote memory over PCl-Express 3.0 (approximately $11 \mathrm{~GB} / \mathrm{s}$ and $25 \mu \mathrm{s}$ ) and NVLink 2.0 (approx. $138 \mathrm{~GB} / \mathrm{s}$ and $10 \mu \mathrm{s}$ ) for all combinations of GPUs on the NVIDIA DGX-2 server. Memory bandwidth and latency to local memory is approximately $800 \mathrm{~GB} / \mathrm{s}$ and $3.5 \mu \mathrm{s}$, respectively, and is on the diagonal of the matrices.
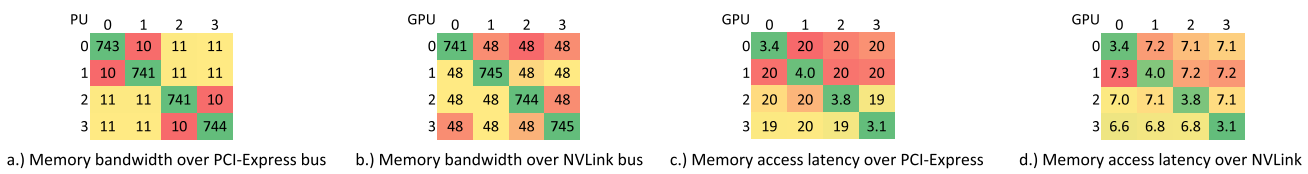

Fig. 12. Comparison of bandwidth and latency for accessing remote memory over PCI-Express 3.0 (approximately 11 GB/s and $20 \mu \mathrm{s}$ ) and NVLink 2.0 (approx. $48 \mathrm{~GB} / \mathrm{s}$ and $7 \mu \mathrm{s}$ ) for all combinations of GPUs on a Barbora node. Memory bandwidth and latency to local memory is approximately $743 \mathrm{~GB} / \mathrm{s}$ and $3.6 \mu$ s, respectively, and is on the diagonal of the matrices.

Table 5. Comparison of Tesla V100, GeForce RTX 2080 Ti and Quadro RTX 8000 NVIDIA GPUs

\begin{tabular}{llll}
\hline GPU card & V100 & 2080 Ti & RTX 8000 \\
\hline Architecture & Volta & Turing & Turing \\
Memory type & HBM2 & GDDR6 & GDDR6 \\
Memory size [GB] & 32 & 11 & 48 \\
Memory throughput [GB/s] $^{\text {[G }}$ & 900 & 616 & 672 \\
NVLink throughput $^{\mathrm{a}}$ [GB/s] & 300 & 100 & 100 \\
Max number of GPUs $_{\text {Number of CUDA Cores }}$ & 16 & 2 & 2 \\
Number of RT cores & 5,120 & 4,352 & 4,608 \\
FP32 performance [TFLOPS] & 15.7 & 68 & 72 \\
\hline
\end{tabular}

${ }^{\text {a}}$ The NVLink throughput is bidirectional.

which uses the Optix plug-in for Blender [Mours 2019], and Cycles with CUDA@Tesla V100. It should be noted that Optix employs RT cores for hardware acceleration of BVH tree traversal.

The results show that hardware acceleration in OptiX 7.0 boosts the performance approximately $2 \times$; see the results for Cycles $w$. OptiX and Cycles w. CUDA, both on GeForce RTX 2080 Ti. The key observation from this test is that Tesla V100 is still comparable to the state-of-the-art GPU, since it gives a better performance than GeForce RTX $2080 \mathrm{Ti}$ for pure CUDA implementation. In the rest of the article, all the results are measured using Cycles with CUDA on Tesla V100. For completeness, we present the key hardware features of both GPUs in Table 5. The parameters of Quadro RTX 8000 are shown in the table, making it the top-of-the-line professional CG GPU card.

\section{GLOBAL SHARED MEMORY PROGRAMMING}

Global shared memory on DGX-2 and Barbora is managed using the Unified Memory mechanism, which creates a unified memory address space of physically separated memory of CPUs and GPUs. It also unifies the memory space of multiple GPUs and their physi- cally separated memory. This greatly simplifies the GPU programming by removing the need for low-level memory management (explicit data transfers between CPU and GPU controlled by a programmer). The main goal of the UM is to provide a consistent view of the data between all CPUs and GPUs within a single server. It ensures that every memory page can be accessed by one CPU or GPU thread at a time. When a GPU accesses a page that is not in its memory, a page fault occurs. The GPU that holds the requested page will release it, and the page will migrate to the GPU that requested it. The same mechanism is used between CPUs and GPUs as well as between GPUs, as thoroughly described in Chien et al. [2020]; Ganguly et al. [2019]; Gayatri et al. [2019].

Memory trashing that continuously migrates the page from one GPU to another easily becomes a serious bottleneck. This has been addressed by hardware support for on-demand page immigration in the Pascal architecture and page access counters in the Volta architecture that enable the frequency of accesses to impact decisions on page migration. For more details, see Sakharnykh [2017a, 2017b].

This problem can also be addressed by a code developer using the data placement and movement hints introduced in CUDA 8.0. These hints are applied using cudaMemAdvise() to a specific part of memory described by the address of the beginning and its size. It the text below, we refer to the part of memory holding data as a chunk of data [NVIDIA 2018b]. The available memory hints are:

- cudaMemAdviseSetReadMostly-communicates that the chunk will be mostly read (but can also be written),

- cudaMemAdviseSetPreferredLocation-sets the location of a chunk to the memory of a given GPU,

- cudaMemAdviseSetAccessedBy-communicates that the chunk will be accessed from a given GPU.

In addition, there is cudaMemPrefetchAsync(), which is used for prefetching (transferring) data to a particular GPU (set by the hints above) before it is used by a GPU kernel. 


\begin{tabular}{|c|c|c|c|}
\hline GPUO & chunk 1 & free memory & \\
\hline GPU1 & chunk 2 & free memory & \\
\hline GPU2 & chunk 3 & free memory & \\
\hline GPU3 & chunk 4 & free memory & \\
\hline
\end{tabular}

a) Fully distributed data structure

chunk 2 chunk 3 chunk 4 free memory

ata structure memory allocation in CPU mempry
cudaMemAdviseSetPreferredLocation

cudaMemAdviseSetReadMostly

Fig. 13. Three ways of allocation of data structure in the Unified Memory of the multi-GPU system.

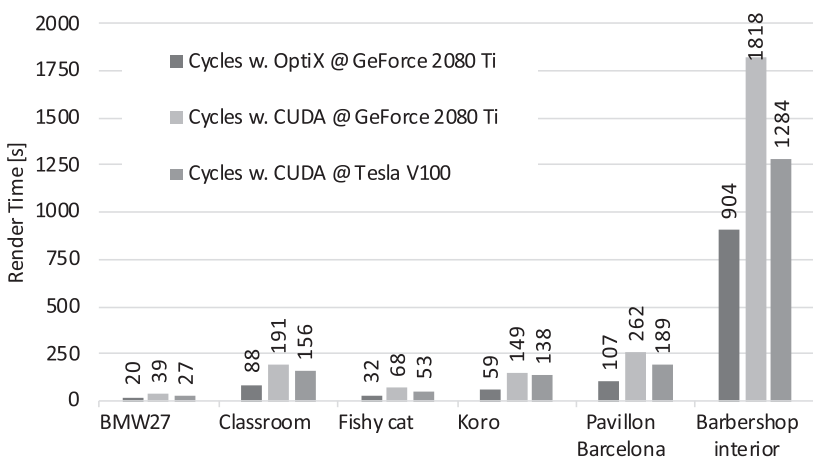

Fig. 14. Comparison of path tracing performance of Blender Cycles on GPU with hardware ray tracing (RT) cores (Cycles w. OptiX 7.0 @ GeForce RTX $2080 \mathrm{Ti}$ ) and Cycles on Tesla V100 GPU installed in NVIDIA DGX-2 machine (Cycles w. CUDA @ Tesla V100).

In our proposed approach the UM is used only among GPUs connected using NVLink bus. Due to low performance of the PCIExpress 3.0 bus connecting CPU and GPU, as shown in Figure 11, the CPU memory is not utilized once data are transferred to GPU memory.

Suppose there is a data structure placed in the UM. If one applies the ...PreferredLocation hint on the entire data structure, then it will be placed in the memory of one GPU. However, different hints can be applied to different chunks of the data structure. This enables us to place different chunks to the memory of different GPUs, i.e., to distribute a data structure across the memories of all GPUs, see Figure 13(a). This is a key principle for processing data structures larger than an individual GPU memory, but it comes with the cost of non-uniform memory access.

If a chunk is marked by the ...ReadMostly hint, then it will be replicated in the memory of all GPUs; see Figure 13(b). This results in optimal performance, as only local memory accesses are performed, but leads to sub-optimal memory utilization.

However, one can also combine the ...PreferredLocation and ...ReadMostly hints and replicate selected chunks of the data structure while distributing the rest of them; see Figure 13(c). This can be used to improve the performance of processing read only data, which is the case of path tracing using the following logic: the pages with a high number of accesses are replicated, and pages with low number of accesses are distributed and owned by the GPU with highest number of accesses.
How to use this simple idea to enable multi-GPU path tracing with distributed scenes is described in Section 4.

\section{ACKNOWLEDGMENTS}

The authors thank Disney for making the Moana Island Scene publicly available. We also thank Alvaro Luna Bautista and Joel Andersdon for the model of Natural History, Three D Scans project for models of statues, and Art Institute of Chicago for free images ( $\mathrm{CC} 0$ ) that have been used in the Museum scene. The Agent scene from the Agent 327: Operation Barbershop movie (agent327.com) is copyright (CC) by Blender Foundation based on original characters (C) by Martin Lodewijk. The Spring scene from Spring movie (cloud.blender.org/films/spring) is copyright (CC) by Blender Foundation. We would like to thank Jaroslav Křivánek for his inspiration and motivation.

\section{REFERENCES}

Neha Agarwal, David Nellans, Mike O'Connor, Stephen W. Keckler, and Thomas F. Wenisch. 2015. Unlocking bandwidth for GPUs in CC-NUMA systems. In Proceedings of the IEEE 21st International Symposium on High Performance Computer Architecture (HPCA'15). IEEE, 354-365.

Timo Aila and Tero Karras. 2010. Architecture considerations for tracing incoherent rays. In Proceedings of the Conference on High Performance Graphics. 113122.

Timo Aila and Samuli Laine. 2009. Understanding the efficiency of ray traversal on GPUs. In Proceedings of the Conference on High Performance Graphics. Association for Computing Machinery, 145-149.

Timo Aila, Samuli Laine, and Tero Karras. 2012. Understanding the Efficiency of Ray Traversal on GPUs-Kepler and Fermi Addendum. NVIDIA Technical Report NVR2012-02. NVIDIA Corporation.

Nabeel Al-Saber and Milind Kulkarni. 2015. SemCache++: Semantics-aware caching for efficient multi-GPU offloading. In Proceedings of the International Conference on Supercomputing. 79-88.

Nabeel AlSaber and Milind Kulkarni. 2013. Semcache: Semantics-aware caching for efficient GPU offloading. In Proceedings of the 27th International ACM Conference on International Conference on Supercomputing. 421-432.

AMD. 2017. AMD EPYC SoC Delivers Exceptional Results on the STREAM Benchmark on 2P Servers. Retrieved from https://www.amd.com/system/files/2017-06/AMD EPYC-SoC-Delivers-Exceptional-Results.pdf.

Atos. 2017. BullSequana X410 E5 Dense GPU-Accelerated Compute Node. Retrieved from https://atos.net/wp-content/uploads/2017/11/FS_BullSequana_X410E5 en1-web.pdf.

Trinayan Baruah, Yifan Sun, Ali Tolga Dinçer, Saiful A. Mojumder, José L. Abellán, Yash Ukidave, Ajay Joshi, Norman Rubin, John Kim, and David Kaeli. 2020. Griffin: Hardware-software support for efficient page migration in multi-GPU systems. In Proceedings of the IEEE International Symposium on High Performance Computer Architecture (HPCA'20). IEEE, 596-609.

Jeremy Birn. 2015. 3dRender.com: Lighting Challenges. Retrieved from http://www 3drender.com/challenges/.

Blender Foundation. 2018. Cycles Open Source Production Rendering. Retrieved from https://www.cycles-renderer.org/.

Brian Budge, Tony Bernardin, Jeff A. Stuart, Shubhabrata Sengupta, Kenneth I. Joy, and John D. Owens. 2009. Out-of-core data management for path tracing on hybrid resources. Comput. Graphics Forum 28, 2 (2009), 385-396. 
Brent Burley, David Adler, Matt Jen-Yuan Chiang, Hank Driskill, Ralf Habel, Patrick Kelly, Peter Kutz, Yining Karl Li, and Daniel Teece. 2018. The design and evolution of Disney's hyperion renderer. ACM Trans. Graphics 37, 3 (2018).

Brent Burley and Dylan Lacewell. 2008. Ptex: Per-face texture mapping for production rendering. Comput. Graphics Forum 27, 4 (2008), 1155-1164.

Steven W. D. Chien, Ivy B. Peng, and Stefano Markidis. 2020. Performance evaluation of advanced features in CUDA unified memory. In Proceedings of the International Conference for High Performance Computing, Networking, Storage, and Analysis (SC'19).

Cameron Christensen, Thomas Fogal, Nathan Luehr, and Cliff Woolley. 2017 Topology-aware image compositing using NVLink. In Proceedings of the IEEE Symposium on Large Data Analysis and Visualization (LDAV'16). 93-94.

Per Christensen, Julian Fong, Jonathan Shade, Wayne Wooten, Brenden Schubert, Andrew Kensler, Stephen Friedman, Charlie Kilpatrick, Cliff Ramshaw, Marc Bannister et al. 2018. RenderMan: An advanced path-tracing architecture for movie rendering. ACM Trans. Graphics 37, 3 (2018).

Robert L. Cook, Loren Carpenter, and Edwin Catmull. 1987. The reyes image rendering architecture. In Proceedings of the 14th Annual Conference on Computer Graphics and Interactive Techniques (SIGGRAPH'87). 95-102.

D. E. Demarle, C. P. Gribble, S. Boulos, and S. G. Parker. 2005. Memory sharing for interactive ray tracing on clusters. Parallel Comput. 31, 2 (2005), 221-242. www. scopus.com Cited By :13.

Luca Fascione, Johannes Hanika, Mark Leone, Marc Droske, Jorge Schwarzhaupt, Tomáš Davidovič, Andrea Weidlich, and Johannes Meng. 2018. Manuka: A batchshading architecture for spectral path tracing in movie production. ACM Trans. Graphics 37, 3 (2018).

Debashis Ganguly, Ziyu Zhang, Jun Yang, and Rami Melhem. 2019. Interplay between hardware prefetcher and page eviction policy in CPU-GPU unified virtual memory. In Proceedings of the International Symposium on Computer Architecture. 224235.

Debashis Ganguly, Ziyu Zhang, Jun Yang, and Rami Melhem. 2020. Adaptive page migration for irregular data-intensive applications under GPU memory oversubscription. In Proceedings of the IEEE International Parallel and Distributed Processing Symposium (IPDPS'20). IEEE, 451-461.

Rahulkumar Gayatri, Kevin Gott, and Jack Deslippe. 2019. Comparing managed memory and ATS with and without prefetching on NVIDIA Volta GPUs. In Proceedings of the IEEE/ACM Performance Modeling, Benchmarking and Simulation of High Performance Computer Systems (PMBS'19). 41-46. DOI : https://doi.org/10. 1109/PMBS49563.2019.00010

Isaac Gelado, John E. Stone, Javier Cabezas, Sanjay Patel, Nacho Navarro, and Wenmei W. Hwu. 2010a. An asymmetric distributed shared memory model for heterogeneous parallel systems. In Proceedings of the 15th International Conference on Architectural Support for Programming Languages and Operating Systems. 347358 .

Isaac Gelado, John E. Stone, Javier Cabezas, Sanjay Patel, Nacho Navarro, and Wenmei W. Hwu. 2010b. An asymmetric distributed shared memory model for heterogeneous parallel systems. In Proceedings of the International Conference on Architectural Support for Programming Languages and Operating Systems (ASPLOS'10). 347-358.

Iliyan Georgiev, Thiago Ize, Mike Farnsworth, Ramón Montoya-Vozmediano, Alan King, Brecht Van Lommel, Angel Jimenez, Oscar Anson, Shinji Ogaki, Eric Johnston et al. 2018. Arnold: A brute-force production path tracer. ACM Trans. Graphics 37, 3 (2018)

Mark Harris. 2017. Unified Memory for CUDA Beginners. Retrieved from https: //devblogs.nvidia.com/unified-memory-cuda-beginners/.

John Hennessy, Mark Heinrich, and Anoop Gupta. 1999. Cache-coherent distributed shared memory: Perspectives on its development and future challenges. Proc. IEEE 87, 3 (1999), 418-429.

Huynh P. Huynh, Andrei Hagiescu, Weng-Fai Wong, and Rick S. M. Goh. 2012. Scalable framework for mapping streaming applications onto multi-GPU systems. ACM SIGPLAN Notices 47, 8 (2012), 1-10.

IT4Innovations. 2019. Barbora supercomputer cluster. Retrieved from https://docs. it4i.cz/barbora/introduction/.

Thomas B. Jablin, James A. Jablin, Prakash Prabhu, Feng Liu, and David I. August. 2012a. Dynamically managed data for CPU-GPU architectures. In Proceedings of the 10th International Symposium on Code Generation and Optimization. 165174

Thomas B. Jablin, James A. Jablin, Prakash Prabhu, Feng Liu, and David I. August. 2012b. Dynamically managed data for CPU-GPU architectures. In Proceedings of the International Symposium on Code Generation and Optimization (CGO'12). 165174

Milan Jaros, Lubomir Riha, Tomas Karasek, Petr Strakos, and Daniel Krpelik. 2017. Rendering in Blender cycles using MPI and Intel Xeon Phi. In Proceedings of the International Conference on Computer Graphics and Digital Image Processing (CGDIP 2017), A. H. Nasri (Ed.). Asia Pacific Institute of Science and Engineering. DOI : https://doi.org/10.1145/3110224.3110236

Florian Kainz, Rod Bogart, and Piotr Stanczyk. 2009. Technical introduction to OpenEXR. Industrial Light Magic (2009), 21.
Toshiaki Kato, Hitoshi Nishimura, Tadashi Endo, Tamotsu Maruyama, Jun Saito, and Per H. Christensen. 2001. Parallel rendering and the quest for realism: The "kilauea" massively parallel ray tracer. In Alan Chalmers, Practical Parallel Processing for Today's Rendering Challenges. SIGGRAPH 2001 Course Note \#40. IV-1 to IV-59.

M. J. Keates and R. J. Hubbold. 1994. Accelerated Ray Tracing on the KSR1 Virtual Shared-Memory Parallel Computer. Citeseer.

Alexander Keller, Carsten Wächter, Matthias Raab, Daniel Seibert, Dietger van Antwerpen, Johann Korndörfer, and Lutz Kettner. 2017. The Iray Light Transport Simulation and Rendering System. Retrieved from https://arxiv:cs.GR/1705.01263.

Youngsok Kim, Jae-Eon Jo, Hanhwi Jang, Minsoo Rhu, Hanjun Kim, and Jangwoo Kim. 2017. GPUpd: A fast and scalable multi-GPU architecture using cooperative projection and distribution. In Proceedings of the Annual International Symposium on Microarchitecture (MICRO'17). 574-586.

Alex Krizhevsky, Ilya Sutskever, and Geoffrey E. Hinton. 2012. ImageNet classification with deep convolutional neural networks. In Advances in Neural Information Processing Systems, Vol. 2. MIT Press, 1097-1105.

Christopher Kulla, Alejandro Conty, Clifford Stein, and Larry Gritz. 2018. Sony pictures imageworks Arnold. ACM Trans. Graphics 37, 3 (2018).

A. Li, S. L. Song, J. Chen, J. Li, X. Liu, N. R. Tallent, and K. J. Barker. 2020. Evaluating modern GPU interconnect: PCIe, NVLink, NV-SLI, NVSwitch and GPUDirect. IEEE Trans. Parallel Distrib. Syst. 31, 1 (2020), 94-110. DOI : https://doi.org/10.1109/ TPDS.2019.2928289

Ang Li, Shuaiwen L. Song, Jieyang Chen, Xu Liu, Nathan Tallent, and Kevin Barker. 2018. Tartan: Evaluating modern GPU interconnect via a multi-GPU benchmark suite. In Proceedings of the IEEE International Symposium on Workload Characterization (IISWC'18). 191-202.

Maxon. 2019. Redshift. Retrieved from https://www.redshift3d.com/product/features\# all.

John D. McCalpin. 1995. Memory bandwidth and machine balance in current high performance computers. IEEE Comput. Soc. Tech. Comm. Comput. Arch. Newslett. (Dec. 1995), 19-25

Steven Molnar, Michael Cox, David Ellsworth, and Henry Fuchs. 1994. A sorting classification of parallel rendering. IEEE Comput. Graph. Appl. 14, 4 (July 1994), 23-32. DOI : https://doi.org/10.1109/38.291528

Patrick Mours. 2019. Accelerating Cycles using NVIDIA RTX. Retrieved from https://code.blender.org/2019/07/accelerating-cycles-using-nvidia-rtx/.

Paul A. Navrátil, Hank Childs, Donald S. Fussell, and Calvin Lin. 2014. Exploring the spectrum of dynamic scheduling algorithms for scalable distributedmemory ray tracing. IEEE Trans. Visual. Comput. Graph. 20, 6 (2014), 893906.

Paul A. Navrátil, Donald S. Fussell, Calvin Lin, and Hank Childs. 2012. Dynamic scheduling for large-scale distributed-memory ray tracing. In Eurographics Symposium on Parallel Graphics and Visualization, Hank Childs, Torsten Kuhlen, and Fabio Marton (Eds.). The Eurographics Association. DOI : https://doi.org/10.2312/ EGPGV/EGPGV12/061-070

NVIDIA. 2017. NVIDIA Tesla V100 GPU Architecture. Retrieved from http://images nvidia.com/content/volta-architecture/pdf/volta-architecture-whitepaper.pdf.

NVIDIA. 2018a. CUDA C Programming Guide. Retrieved from https://docs.nvidia. com/cuda/archive/10.0/pdf/CUDA_C_Programming_Guide.pdf.

NVIDIA. 2018b. CUDA Runtime API. Retrieved from https://docs.nvidia.com/cuda/ archive/10.0/pdf/CUDA Runtime API.pdf.

NVIDIA. 2018c. NVIDIA NVSWITCH Technical Overview. Retrieved from https:// images.nvidia.com/content/pdf/nvswitch-technical-overview.pdf.

NVIDIA. 2018d. NVIDIA Turing GPU Architecture. Retrieved from https:// www.nvidia.com/content/dam/en-zz/Solutions/design-visualization/ technologies/turing-architecture/NVIDIA-Turing-Architecture-Whitepaper.pdf.

NVIDIA. 2019. DGX-2/2H SYSTEM User Guide. Retrieved from https://docs.nvidia com/dgx/pdf/dgx2-user-guide.pdf. DU-09130-001_v08.1.

Jacopo Pantaleoni, Luca Fascione, Martin Hill, and Timo Aila. 2010. PantaRay: Fast ray-traced occlusion caching of massive scenes. ACM Trans. Graphics 29, 4 (JUL 2010). DOI : https://doi.org/10.1145/1778765.1778774

Steven Parker, William Martin, Peter-Pike J. Sloan, Peter Shirley, Brian Smits, and Charles Hansen. 1999. Interactive ray tracing. In Proceedings of the Symposium on Interactive 3D Graphics. 119-126.

Steven Parker, Peter Shirley, Yarden Livnat, Charles Hansen, and Peter-Pike Sloan. 1998. Interactive ray tracing for Ssosurface rendering. In Proceedings of the IEEE Visualization Conference. 233-238.

PIXAR. 2019. OpenSUBDIV. Retrieved from http://graphics.pixar.com/opensubdiv/.

Jelica Protic, Milo Tomasevic, and Veljko Milutinovic. 1995. A survey of distributed shared memory systems. In Proceedings of the 28th Annual Hawaii International Conference on System Sciences, Vol. 1. IEEE, 74-84.

Jelica Protic, Milo Tomasevic, and Veljko Milutinovic. 1996. Distributed shared memory: Concepts and systems. IEEE Parallel Distrib. Technol.: Syst. Appl. 4, 2 (1996), $63-71$.

Amit Sabne, Putt Sakdhnagool, and Rudolf Eigenmann. 2013. Scaling large-data computations on multi-GPU accelerators. In Proceedings of the International Conference on Supercomputing. 443-454. 
Nikolay Sakharnykh. 2017a. Maximizing Unified Memory Performance in CUDA. Retrieved from https://devblogs.nvidia.com/maximizing-unified-memoryperformance-cuda/.

Nikolay Sakharnykh. 2017b. Unified memory on Pascal and Volta. In Proceedings of the GPU Technology Conference (GTC'17). Retrieved from http://on-demand.gputechconf.com/gtc/2017/presentation/s7285-nikolaysakharnykh-unified-memory-on-pascal-and-volta.pdf.

Irani Sarosh. 2019. Accelerated computing solutions for AI and HPC workloads. Retrieved from https://developer.nvidia.com/gtc/2019/video/S9981. In Proceedings of the GPU Technology Conference (GTC'19).

Hyunseok Seo, Jinwook Kim, and Min-Soo Kim. 2015. GStream: A graph streaming processing method for large-scale graphs on GPUs. In Proceedings of the ACM SIGPLAN Symposium on Principles and Practice of Parallel Programming (PPOPP'15). 253-254.

Hideyuki Shamoto, Koichi Shirahata, Aleksandr Drozd, Hitoshi Sato, and Satoshi Matsuoka. 2015. Large-scale distributed sorting for GPU-based heterogeneous supercomputers. In Proceedings of the IEEE International Conference on Big Data (IEEEBigData'14). 510-518.

J. Pal Singh, Anoop Gupta, and Marc Levoy. 1994. Parallel visualization algorithms: Performance and architectural implications. Computer 27, 7 (1994), 45-55.

Myungbae Son and Sung-Eui Yoon. 2017. Timeline scheduling for out-of-core ray batching. In Proceedings of the Conference on High Performance Graphics (HPG'17).

Vijayaraghavan Soundararajan, Mark Heinrich, Ben Verghese, Kourosh Gharachorloo, Anoop Gupta, and John Hennessy. 1998. Flexible use of memory for replication/migration in cache-coherent DSM multiprocessors. In Proceedings of the 25th Annual International Symposium on Computer Architecture. IEEE, 342-355.

Blender Studio. 2020a. Agent 327-Blender Cloud. Retrieved from https://cloud. blender.org/films/agent-327.

Blender Studio. 2020b. Spring-Blender Cloud. Retrieved from https://cloud.blender. org/films/spring.

The Art Institute of Chicago. 2020. Discover Art \& Artists. Retrieved from https:// www.artic.edu/collection.
Threedscans. 2020. Three D Scans. Retrieved from https://threedscans.com.

Will Usher, Ingo Wald, Jefferson Amstutz, Johannes Gunther, Carson Brownlee, and Valerio Pascucci. 2019. Scalable ray tracing using the distributed FrameBuffer. Computer Graphics Forum 38, 3 (2019), 455-466. DOI : https://doi.org/10.1111/cgf 13702arXiv:https://onlinelibrary.wiley.com/doi/pdf/10.1111/cgf.13702

I. Wald, C. Benthin, and P. Slusallek. 2003. Distributed interactive ray tracing of dynamic scenes. In Proceedings of the IEEE Symposium on Parallel and Large-Dato Visualization and Graphics (PVG'03). 77-85.

Ingo Wald, Sven Woop, Carsten Benthin, Gregory S. Johnson, and Manfred Ernst. 2014. Embree: A kernel framework for efficient CPU ray tracing. ACM Trans. Graphics 33, 4 (2014), 1-8.

Walt Disney Animation Studios. 2018. Moana Island Scene. Retrieved from https:// www.disneyanimation.com/resources/moana-island-scene/.

Rui Wang, Yuchi Huo, Yazhen Yuan, Kun Zhou, Wei Hua, and Hujun Bao. 2013. GPU-based out-of-core many-lights rendering. ACM Trans. Graphics 32, 6 (2013).

Chenhao Xie, Fu Xin, Mingsong Chen, and Shuaiwen L. Song. 2019. OO-VR: NUMA friendly object-oriented VR rendering framework for future NUMA-based multiGPU systems. In Proceedings of the International Symposium on Computer Architecture. 53-65.

Vinson Young, Aamer Jaleel, Evgeny Bolotin, Eiman Ebrahimi, David Nellans, and Oreste Villa. 2018. Combining HW/SW mechanisms to improve NUMA performance of multi-GPU systems. In Proceedings of the 51st Annual IEEE/ACM International Symposium on Microarchitecture (MICRO'18). IEEE, 339351.

Kun Zhou, Qiming Hou, Zhong Ren, Minmin Gong, Xin Sun, and Baining Guo. 2009. RenderAnts: Interactive REYES rendering on GPUs. ACM Trans. Graphics 28, 5 (Dec. 2009). DOI : https://doi.org/10.1145/1618452.1618501 ACM SIGGRAPH Asia Conference 2009, Yokohama, JAPAN, DEC 16-19, 2009.

Received September 2020; revised December 2020; accepted January 2021 